\title{
The space infrared telescope for cosmology and astrophysics: SPICA A joint mission between JAXA and ESA
}

\author{
Bruce Swinyard • Takao Nakagawa
}

Received: 31 October 2007 / Accepted: 21 March 2008/Published online: 13 May 2008

(C) Springer Science + Business Media B.V. 2008

\begin{abstract}
The Space Infrared telescope for Cosmology and Astrophysics (SPICA) is planned to be the next space astronomy mission observing in the infrared. The mission is planned to be launched in 2017 and will feature a $3.5 \mathrm{~m}$ telescope cooled to $<5 \mathrm{~K}$ through the use of mechanical coolers. These coolers will also cool the focal plane instruments thus avoiding the use of consumables and giving the mission a long lifetime. SPICA's large, cold aperture will provide a two order of magnitude sensitivity advantage over current far infrared facilities ( $>30$ microns wavelength). We describe the scientific advances that will be made possible by this large increase in sensitivity and give details of the mission, spacecraft and focal plane conceptual design.
\end{abstract}

Keywords Space missions $\cdot$ Infrared

Co-authored by the SPICA Consortium:

Patrick Merken (IMEC/RMA), Pierre Royer (KUL), Tim Souverijns (IMEC/RMA), Bart

Vandenbussche (KUL), Christoffel Waelkens (KUL), Peter Davis (BSS), James Di Francesco (HIA/NRC), Mark Halpern (UBC), Martin Houde (UWO), Doug Johnstone (HIA/NRC), Gilles Joncas (Université Laval), David Naylor (Leth), Rene Plume (Calg), Douglas Scott (UBC), Abergel, A. (IAS), Bensammar, S. (GEPI), Braine, J. (LAB), Buat, V. (OAMP), Burgarella, D. (OAMP), Cais, Ph. (LAB), Dole, H (IAS), Duband, L. (SBT), Elbaz, D. (SAP), Gerin, M. (LERMA), Giard, M. (CESR), Goicoechea, J. (LERMA), Joblin, C. (CESR), Jones, A. (IAS), Kneib, J. P. (OAMP), Lagache, G. (IAS), Madden, S. (SAP), Pons, R. (CESR), Pajot, F. (IAS), Rambaud, D. (CESR), Ravera, L. (CESR), Ristorcelli, I. (CESR), Rodriguez, L. (SAP), Vives, S. (OAMP), Zavagno, A. (OAMP), Norbert Geis (MPE), Oliver Krause (MPIA), Dieter Lutz (MPE), Albrecht Poglitsch (MPE), Walfried Raab, (MPE), Jutta Stegmaier (MPIA), Eckhard Sturm (MPE), Richard Tuffs (MPIK), Hyung Mok Lee (SNU), Bon-Chul Koo (SNU), Myungshin Im (SNU), Soojong Pak (KHU), Wonyong Han (KASI), Jang-Hyun Park (KASI), Uk-Won Nam (KASI), Ho Jin (KASI), Dae-Hee Lee (KASI), In-Soo Yuk (KASI), Sungho Lee (KASI), Yuri Aikawa (Kobe), Nobuo Arimoto (NAOJ), Yasuo Doi, (Tokyo), Keigo Enya (JAXA), Misato Fukagawa (Nagoya), Reiko Furusho (NAOJ), Sunao Hasegawa (JAXA), Masahiko Hayashi (NAOJ), Mitsuhiko Honda (Kanagawa), Shigeru Ida (TInstT), Masatoshi Imanishi (NAOJ), Shu-ichiro Inutsuka (Kyoto), Hideyuki Izumiura (NAOJ), Hideyuki Kamaya (Kyoto), Hidehiro Kaneda (JAXA), Toshihiro Kasuga (NAOJ), Hirokazu Kataza (JAXA), Koji Kawabata (Hiroshima), Mitsunobu Kawada (Nagoya), Hideyo Kawakita (KSU), Tsuneo Kii (JAXA), Jin Koda (Caltech), Tadayuki Kodama (NAOJ), Eiichiro Kokubo (NAOJ), Keiji Komatsu (JAXA), Hideo Matsuhara (JAXA), Toshio Matsumoto (JAXA), Shuji Matsuura (JAXA), Takashi Miyata (Tokyo), Takashi Miyata (Tokyo), Hiroshi Murakam (JAXA), Hirohisa Nagata (JAXA), Tetsuya Nagata (Kyoto), Tadashi Nakajima (NAOJ), Kobayashi Naoto (Tokyo), Ryoichi Nishi (Niigata), Atsushi Noda (JAXA), Atsushi Okamoto (JAXA), Yoshiko K. Okamoto (Ibaraki), Kazuyuki Omukai (NAOJ), Takashi Onaka (Tokyo), Takafumi Ootsubo (Nagoya), Masami Ouchi (STScI), Hirobumi Saito (JAXA), Yoichi Sato (JAXA), 
Shigeyuki Sako (Tokyo), Tomohiko Sekiguchi (NAOJ), Hiroshi Shibai (Nagoya), Hiroyuki Sugita (JAXA), Koji Sugitani (Nagoya), Hajime Susa (Rikkyo University), Pyo Tae-soo (NAOJ), Motohide Tamura (NAOJ), Yoshihiro Ueda (Kyoto), Munetaka Ueno (Tokyo), Takehiko Wada (JAXA), Jun'ichi Watanabe (NAOJ), Toru Yamada (NAOJ), Issei Yamamura (JAXA), Naoki Yoshida (Nagoya), Kitamura Yoshimi (JAXA), Yukari Yui (JAXA), Milena Benedettini (IFSI), Riccardo Cerulli (IFSI), Anna Di Giorgio (IFSI), Sergio Molinari (IFSI), Renato Orfei (IFSI), Stefano Pezzuto (IFSI), Lorenzo Piazzo (La Sapienza), Paolo Saraceno (IFSI), Luigi Spinoglio (IFSI), Thijs de Graauw (LO), Piet de Korte (SRON), Frank Helmich (SRON), Henk Hoevers (SRON), Robert Huisman (SRON), Russell Shipman (SRON), Floris van der Tak (SRON/ Groningen), Paul van der Werf (LO), Wolfgang Wild (SRON), Jose Acosta-Pulido (IAC), Jose Cernicharo (CSIC), Jose Herreros (IAC), Jesus Martin-Pintado (CSIC), Francisco Najarro (CSIC), Ismael PerezFourmon (IAC), Juan Ramon Pardo (CSIC), Francisca Gomez (CSIC), Nieves Castro Rodriguez (CSIC), Peter Ade (CU), Mike Barlow (UCL), David Clements (ICL), Marc Ferlet (RAL), Helen Fraser (Strathclyde), Douglas Griffin (RAL), Matthew Griffin (CU), Peter Hargrave (CU), Kate Isaak (CU), Robert Ivison (ATC), Malik Mansour (RAL), Jonathan Laniesse (RAL), Phillip Mauskopf (CU), Dmitry Morozov (CU), Seb Oliver (Sussex University), Angiola Orlando (CU), Mathew Page (UCL-MSSL), Cristina Popescu (UCLAN), Stephen Serjeant (OU), Rashmi Sudiwala (CU), Dimitra Rigopoulou (Oxford), Ian Walker (CU), Glenn White, (OU/RAL), Serena Viti (UCL), Berend Winter (UCL-MSSL), Jamie Bock (JPL), Matt Bradford (JPL), Martin Harwit (Cornell), Warren Holmes (JPL)

BSS-BlueSky Spectroscopy, Inc., Lethbridge, Canada

Caltech-California Institute of Technology, USA

CU-Cardiff University, UK

SBT-CEA-CENG, Service des Basses Températures, Grenoble, France

SAP_CEA-DAPNIA, Service d'Astrophysique, Saclay, France

CESR - Centre d'Etude Spatiale des Rayonnements, Université Toulouse 3-CNRS, France

Cornell-Cornell University, USA

CSIC_-Departamento de AstrofÃsica Molecular e InfraRoja, IEM, CSIC, Madrid, Spain

GEPI-GEPI, CNRS-Observatoire de Paris, France

Groningen-Groningen University, The Netherlands

Gunma Astronomical Observatory, Japan

HIA/NRC - Herzberg Institute for Astrophysics, Canada

Hiroshima-Hiroshima University, Japan

Ibaraki-Ibaraki University, Japan

IMEC/RMA, Belgium

ICL-Imperial College, London, UK

IAP - Institut d'Astrophysique de Paris, CNRS, Paris, France

IAS - Institut d'Astrophysique Spatiale, CNRS-Paris XI, Orsay, France

IAC - Instituto de Astrofísica de Canarias, Spain

KUL-Instituut voor Sterrenkunde, Katholieke Universiteit, Leuven, Belgium

IFSI-Istituto di Fisica dello spazio Interplanetario, Italy

JAXA_ Japan Aerospace Exploration Agency, Japan

JPL_-Jet Propulsion Laboratory, USA

KASI-Korea Astronomy and Space Science Institute, Daejon, Korea

Kobe-Kobe University, Japan

KSU-Kyoto Sangyo University, Japan

Kyoto-Kyoto University, Japan

KHU_Kyung Hee University, Korea

LAB - Laboratoire d'Astrophysique de Bordeaux, CNRS-OASU, Bordeaux, France

LAM-Laboratoire d'Astrophysique de Marseille, CNRS-OAMP, Marseille, France

LO_-Leiden Observatory, University of Leiden, The Netherlands

LERMA, CNRS-Observatoire de Paris, France

MPIA-Max-Planck-Institut für Astronomie, Germany

MPE-Max-Planck-Institut für extraterrestrische Physik, Germany

MPIK-Max-Planck-Institut für Kernphysik, Germany

UCL-MSSL_-Mullard Space Science Laboratory, UK

Nagoya-Nagoya University, Japan

Nagoya City University, Japan

NAOJ_-National Astronomical Observatory of Japan, Japan

SRON_Netherlands Institute for Space Research, The Netherlands 


\section{Introduction and SPICA in Context}

Since the advent of observational astronomy in the Mid-Infrared (MIR) and FarInfrared (FIR) wavelength range with Infrared Astronomical Satellite (IRAS) [1] and the Kuiper Airborne Observatory [2], and after the success of the infrared space observatories (ISO) Spitzer and AKARI [3-5], it has become increasingly clear that we can only fully understand the evolution of the Universe and of all its constituent parts by observing the MIR/FIR emission from dust and gas. In order to properly observe between $\sim 5$ and $210 \mu \mathrm{m}$ it is necessary to use spaceborne infrared telescopes as ground based or even airborne observatories can only cover restricted waveband ranges at limited sensitivities. It is also necessary that any MIR/FIR observatory is cooled to temperatures at which the self-emission from the telescope and instruments does not overwhelm the signal from astronomical sources. To give a low ambient photon background across the whole MIR/FIR waveband requires a telescope temperature less than $\sim 5 \mathrm{~K}$, and all space observatories to date (IRAS, ISO, Spitzer and AKARI) have used liquid helium (LHe) to cool their telescopes and instruments to below $\sim 5 \mathrm{~K}$. This has necessitated the use of large quantities of liquid cryogen to cool the telescopes, resulting in aperture sizes of less than $1 \mathrm{~m}$. To date, therefore, our ability to characterise the cold and dusty Universe has been limited by poor spatial resolution and low sensitivity relative to other wavelength ranges, despite the development of highly sensitive detectors, especially in the 5-30 $\mu \mathrm{m}$ band.

\footnotetext{
Niigata University, Japan

Rikkyo University, Japan

RAL-Rutherford Appleton Laboratory, UK

STScI-Space Telescope Science Institute, USA

SNU-Seoul National University, Seoul, Korea

Strathclyde University, UK

Sussex University, UK

OU-The Open University, UK

TInstT-Tokyo Institute of Technology, Japan

ATC - UK Astronomy Technology Centre, Edinburgh, UK

Università di Roma, Italy

UBC-University of British Columbia, Canada

Calg-University of Calgary, Canada

UCL_University College, London, UK

UCLAN-University of Central Lancashire, Preston, UK

Université Laval, Quebec, Canada

Leth-University of Lethbridge, Canada

Oxford-University of Oxford, UK

Tokyo-University of Tokyo, Japan

UWO-University of Western Ontario, Canada
}

B. Swinyard $(\bowtie)$

Science and Technology Facilities Council, Rutherford Appleton Laboratory, Chilton, Didcot,

Oxfordshire OX11 0QX, UK

e-mail: b.m.swinyard@rl.ac.uk 
This situation will be improved in the coming decade with the advent of the Herschel Space Observatory [6] with an 80-K 3.5 m telescope operating from 57 to $700 \mu \mathrm{m}$ and the James Webb Space Telescope (JWST) [7] with a 45-K, 25-m ${ }^{2}$ telescope operating from 0.6 to $28 \mu \mathrm{m}$ and diffraction limited at $2 \mu \mathrm{m}$ and beyond. Herschel and JWST will represent a leap in our ability to observe in the MIR/FIR and, with JWST at least, come close to the spatial resolution and sensitivity limits obtained in the optical and sub-mm bands. However, both have drawbacks in terms of advancing our full understanding of the MIR/FIR Universe: JWST does not cover wavelengths longer than $28 \mu \mathrm{m}$; Herschel only starts coverage at $57 \mu \mathrm{m}$ and, because of its relatively warm aperture, Herschel only gives a modest improvement in sensitivity compared to Spitzer, despite its superior imaging and spectroscopic capabilities.

To break through the sensitivity limitations of Herschel and the narrow wavelength coverage of JWST, the Japanese space agency (Institute of Space and Astronautical Science (ISAS)/Japan Aerospace Exploration Agency (JAXA)) is proposing to build Space Infrared telescope for Cosmology and Astrophysics (SPICA). This mission can be built within 10 years without major technical development, but will offer a breakthrough in sensitivity in the FIR region and will provide MIR capabilities to cover the MIR/FIR waveband within a single facility. SPICA will have a $3.5 \mathrm{~m}$ telescope actively cooled to below $5 \mathrm{~K}$ to remove all self emission, thus massively increasing the potential sensitivity in the FIR compared with Herschel. Furthermore, SPICA will employ mechanical coolers rather than cryogens giving it a long lifetime whilst still allowing a large mirror size. The telescope will be monolithic, like Herschel, and will in principle have a much cleaner point spread function than a deployable segmented mirror, allowing diffractionlimited performance across the full wavelength range. To take advantage of this lowbackground environment the instrument suite proposed will have wide field MIR and FIR imaging, medium to high spectral resolution MIR and FIR spectroscopy and a MIR coronagraph designed for imaging and spectroscopy of young Jupiter-like planets around nearby stars.

In order to place the potential performance of the SPICA mission in context, Figs. 1 and 2 show the wavelength coverage and estimated point source sensitivities for photometric and spectroscopic observations that will be possible with SPICA. Also shown in the same figures are the sensitivities of the predecessor (Herschel) and complementary facilities such as JWST [8] and Atacama Large Millimeter Array (ALMA) [9] that will be in operation in the same time. In preparing these figures we have used publicly available sensitivities for Herschel, JWST and ALMA and have calculated the SPICA sensitivities using the same model formulation as used for JWST [8] and Herschel [10]. For the MIR range $(5-37 \mu \mathrm{m})$ we assume state of the art performance (SOAP) Si:Sb or Si:As detectors as used on Spitzer and JWST. For the 35-210 $\mu \mathrm{m}$ wavelength range two possibilities are shown: SOAP performance based on a combination of Ge:Ga photoconductors and a development of the Herschel-PACS bolometer arrays; and Goal performance based on the development of Transition Edge Superconducting (TES) bolometer arrays based on devices used for the Submillimetre Common-User Bolometer Array (SCUBA)-2 instrument on the JCMT [11] and development programmes ongoing in European institutes [12]. The basic line sensitivity is calculated for two cases: an FTS with three bands of $R=$ 
Fig. 1 Predicted photometric performance of SPICA (green and purple) compared to predecessor and complementary facilities (red) given as point source sensitivities in microJansky for $5-\sigma$ in $1 \mathrm{~h}$ over the bands shown indicatively as horizontal lines. Note the two orders of magnitude increase in FIR photometric sensitivity compared to PACS that will be achieved using goal sensitivity detectors on SPICA. The figures here are raw sensitivity only. The effects of confusion, and their mitigation, are discussed later

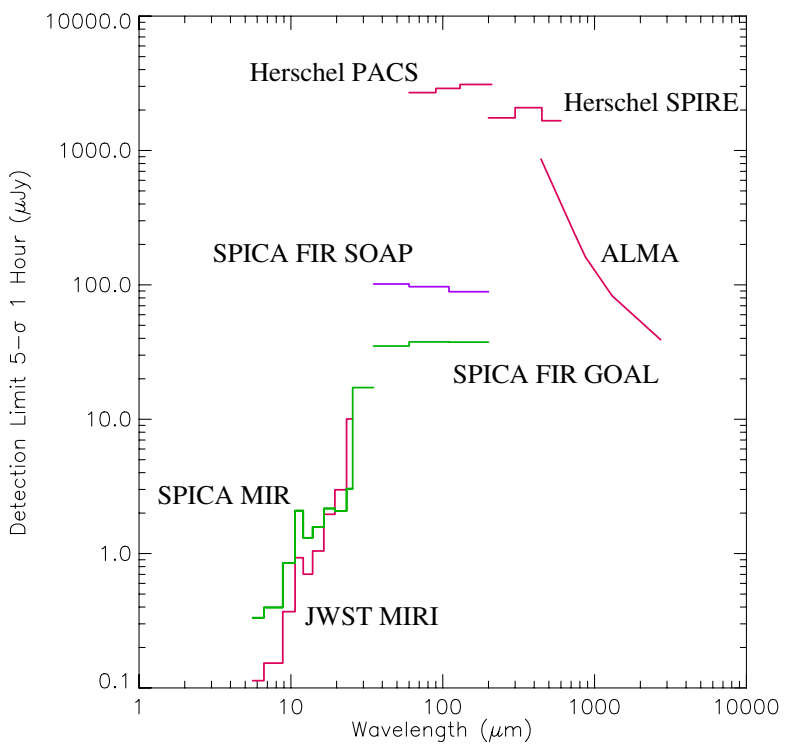

3 (solid lines in Fig. 2) and a possible alternative design with band limiting 1\% filtering with an overall lower throughput (dashed lines in Fig. 2). To take full advantage of an increase in detector performance some form of band limiting will be required.

It is clear from Figs. 1 and 2 that a huge gain in sensitivity over Herschel can be achieved with SPICA even with SOAP detectors, especially when combined with

Fig. 2 Predicted spectroscopic performance of SPICA (green and purple) compared to predecessor and complementary facilities (red) given as single unresolved line sensitivity for a point source in watt per meter squared for 5-s in $1 \mathrm{~h}$. For ALMA $100 \mathrm{~km} / \mathrm{s}$ resolution is assumed as this is appropriate for extra-galactic sources. The SPICA MIR sensitivities are scaled by telescope area from the JWST and Spitzer IRS values respectively. The dashed lines are for a band limited FTS $(R \sim$ $100)$ with $1 / 2$ the throughput of the full band instrument

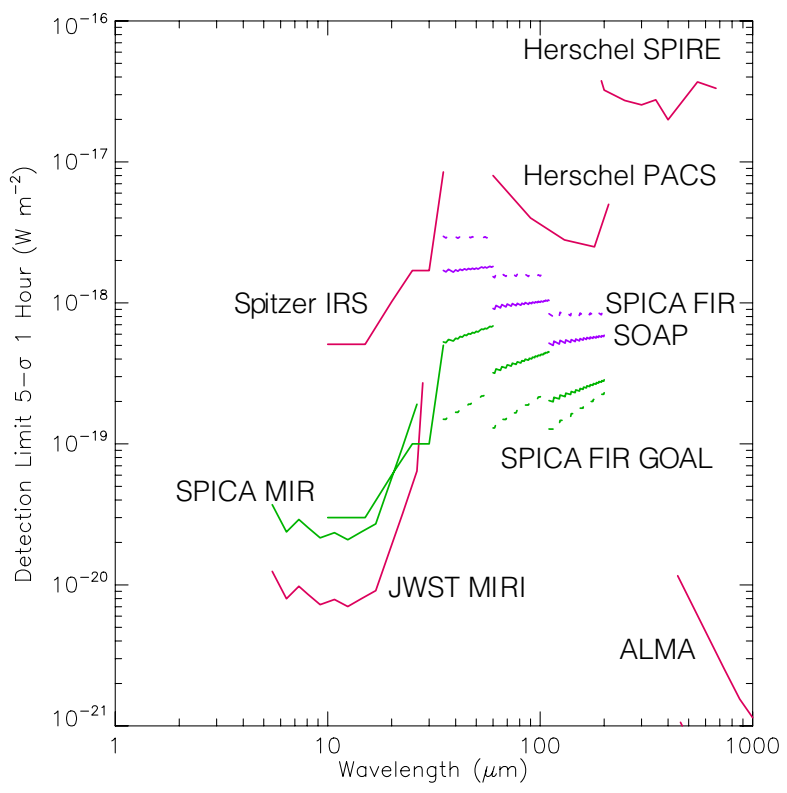


the proposed large fields of view. With a well structured detector development programme it will be possible to deliver detectors with a far better performance and a commensurate leap in detection sensitivity that will, for the first time, place FIR observations in the same league as the sub-millimetre and MIR. In the MIR SPICA will have virtually the same sensitivity as the JWST but over a wider wavelength range enabling it to provide complementary instrument capabilities in the form of a wide field low/medium resolution $(R \sim 200)$ objective grism spectrometer, much higher spectral resolution $(R \sim 30,000)$ to allow the gas dynamics of protoplanetary disks to be examined and an advanced coronagraph that will allow both imaging and spectroscopy of young gas giant planets in hundreds of nearby stars. In the next section we briefly describe the scientific breakthroughs that these major advances in MIR/FIR capability will make possible, in the rest of the paper we describe the mission, spacecraft and proposed instrument suite in more detail.

\section{Science with SPICA}

\subsection{The importance of the MIR/FIR}

The 5-210 $\mu \mathrm{m}$ spectral range is one of the richest windows of the electromagnetic spectrum emitted by astrophysical objects. It spans the wavelength range over which the largest proportion of the energy is emitted during the evolution of galaxies. It plays host to a variety of atomic/ionic forbidden transitions that, spanning a large range in excitation, ionization and density conditions, are among the best to trace gas excited from both stellar and planetary evolution processes as well as non-thermal processes. Radiation in the MIR/FIR, unlike the near infrared (NIR), optical and ultraviolet (UV) light, is largely unaffected by the effects of dust extinction and includes many of the most prominent Polycyclic Aromatic Hydrocarbon (PAH) and silicate features and so can be used to trace star and planet formation as well as active galactic nucleus (AGN) activity in both the local and the very distant Universe. It is also the range in which warm molecular gas found in AGN tori, shocks, stellar envelopes, X-ray illuminated regions, outflows and dense protostellar cores can be studied through the transitions of $\mathrm{CO}, \mathrm{OH}, \mathrm{H}_{2} \mathrm{O}$ and molecular hydrogen. As one moves to higher redshift, the UV/optical-NIR waveband shifts into the MIR/FIR, providing access to rest frame UV-optical spectroscopy which in turn traces the first phases of galaxy evolution through its main constituents: stars and accreting black holes.

\subsection{Planetary system formation: from gas and dust to planets; from ices to oceans}

The study of star formation in our own Galaxy has been revolutionised by spectroscopic observations in the MIR/FIR, as it is only in this waveband that the gas chemistry and gas/grain processes within the highly obscured stellar nurseries can be observed. The spectrometers on Herschel are expected to make further strides in our understanding of star formation but, to date, there has been little or no direct study of the role of MIR/FIR cooling lines in the formation of planetary systems, despite the obvious importance of, for instance, oxygen chemistry to the emergence 
of life. SPICA will have the sensitivity to detect the lines from species such as $\mathrm{O}$, $\mathrm{OH}$ and $\mathrm{H}_{2} \mathrm{O}$ (both gas and ice) from proto-planetary gas disks in the early stages of planetary formation. In fact SPICA will have sufficient sensitivity to detect them in a volume sufficiently large to make the first unbiased study of the chemistry of disks around all spectral types of stars. The huge increase in photometric sensitivity that SPICA will achieve in the FIR will also enable us to trace the presence of cool, dusty disks out to the furthest reaches of the Galaxy, answering directly questions over planetary formation as function of stellar type and age and providing a comprehensive inventory of stars with circumstellar debris disks that will be invaluable to future planet imaging facilities. In the nearest objects with circumstellar disks (such as Vega), SPICA's unique access to the FIR water ice features [15] will allow us to map the "snowline" for the first time (see Fig. 4), giving a critical insight into the role of water ice in the formation and evolution of planetary systems [14].

Gas disks Most young stars are surrounded by accretion disks which are the likely progenitors of planetary systems. The physical and chemical conditions in protoplanetary disks set the boundary conditions for planet formation and an understanding of the formation and evolution of protoplanetary disks will finally link star formation and planetary science. Our current view of these objects states that disks evolve from gas-rich structures where only $\sim 1 \%$ of the total mass is in the form of dust grains, to optically thin debris disks almost completely devoid of gas. Although the dust is relatively easily detected from photometric observations in the 20-100 $\mu \mathrm{m}$ range, very little is known about the gas phase. With the high sensitivity of SPICA over this wavelength region we will be able to detect the line emission from hundreds of proto-planetary gas disks, producing an extensive census of stellar and disk types that will give a critical insight into the conditions under which planets form and the conditions for life emerge. For the strongest lines in the 5-15 $\mu \mathrm{m}$ range, and at spot wavelengths at 17 and $28 \mu \mathrm{m}$ to include all the $\mathrm{H}_{2}$ ground state ortho and para rotation lines, SPICA will have a very high resolution spectrometer $(R \sim 30,000)$ capable of resolving Keplerian rotation to less than $10 \mathrm{~km} / \mathrm{s}$ in the line profiles, so characterising the physical and chemical parameters of gas disks as a function of radius at least in the inner parts of the disk where gas giants may form.

Dust in circumstellar disks The ability of FIR spectroscopy to determine the mineral makeup of dusty disks in young stars is illustrated by the ISO spectrum of HD142527 shown in Fig. 3. SPICA will be 100's of times more sensitive than ISO and so will see not only the young opaque objects like this, but will be able to trace the mineralogy of dust within disks at all stages of planetary system formation and, because of its full wavelength coverage, will probe both the cold and warm dust populations and emission features. SPICA will not only be able to determine the detailed mineralogy of the dust, but will also trace the variation in grain size distribution and temperature, which are both expected to evolve with disk age leading to a variation in the disk SED. SPICA's high photometric sensitivity will allow the detection of as little as $10^{-4}$ Lunar mass of dust at $\sim 100 \mathrm{~K}$ out to tens of parsecs; this is approximately the mass of our own Zodiacal cloud. In summary, 
Fig. 3 The ISO spectrum of the young star HD 142527 [13] showing the model components including the crystalline water ice features. Water ice can only be directly detected at these temperatures through the 44 and $63 \mu \mathrm{m}$ emission features. SPICA will be able to take the equivalent spectra of objects at flux levels of $\sim 1 \mathrm{mJy}$ in $1 \mathrm{~h}$

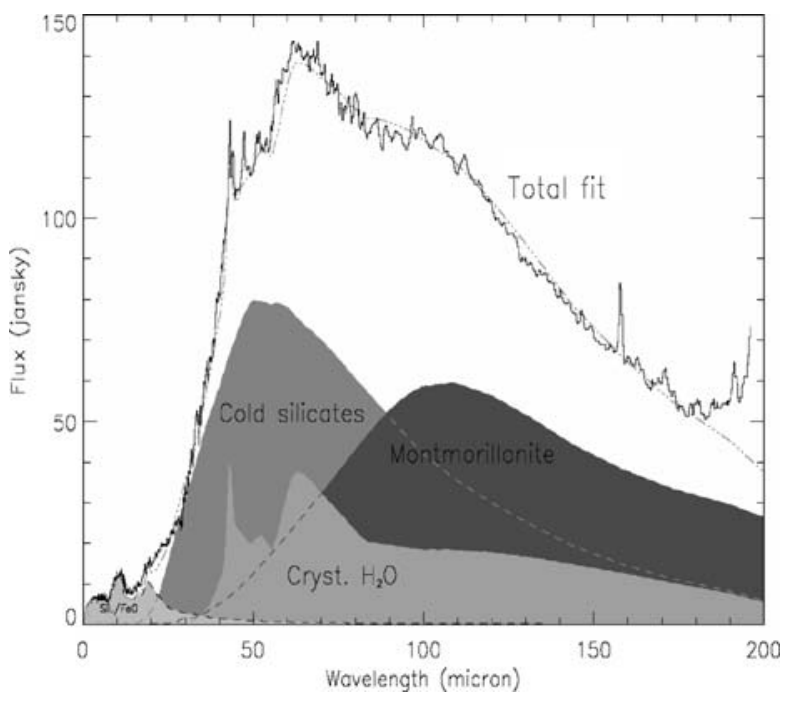

using the full capabilities of SPICA we will reveal for the first time the detailed processes that lead eventually to the formation of Earth-like planets.

In nearby systems we will be able to image disks directly (see Fig. 4) to examine their structure. In the MIR/FIR we will be able to trace the variation in dust mineral content, size distribution and temperature and so, in a few examples, we will be able to directly trace the mineral content and grain size distribution as a function of radius to compare these, together with the spectra of comets, asteroids and TNOs within our own Solar system, with the 100 s of spatially unresolved objects which SPICA

Fig. 4 CSO SHARC II $350 \mu \mathrm{m}$ image of Vega [16] overplotted with the pixel scale of SPICA at 44 to $63 \mu \mathrm{m}$ (white squares). The spatial resolution is equivalent to $\sim 23 \mathrm{AU}$ at this distancethis is sufficient to detect the presence of the expected snowfree region of $42 \mathrm{AU}$ in diameter

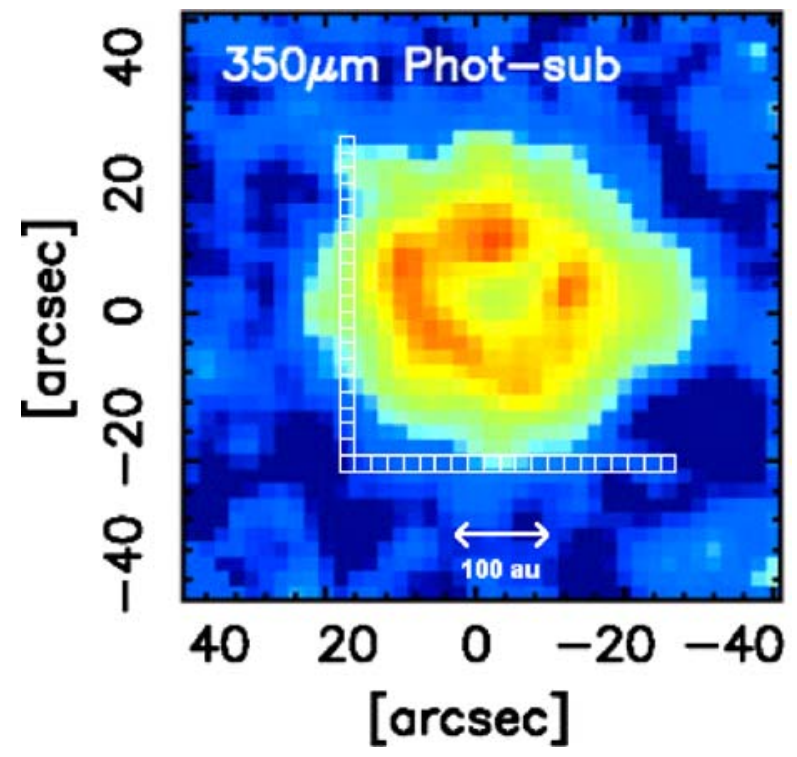


will be able to observe. In more distant stars SPICA will be able to use MIR coronagraphy to image and take spectra of the inner parts of many more disks. It will also be able to take the spectrum of the dust across the full MIR/FIR waveband.

Exo-planets The clean point spread function from SPICA's un-segmented high optical quality telescope will allow a coronagraph operating from $\sim 3$ to $27 \mu \mathrm{m}$ to directly image and, for the first time, take MIR spectra of young gas giant planets that are within 1 Gyr of formation looking for characteristic features of methane, water and ammonia that are tracers of pre-biotic activity. The ability of the coronagraph instrument to detect planets close to the host star will be greatly improved by extending the wavelength range to $3.5 \mu \mathrm{m}$, as this allows coverage of the predicted $4 \mu \mathrm{m}$ emission feature $[17,18]$. We estimate that with a binary mask type coronagraph we will be able to achieve a contrast of $10^{-6}$ at an inner working angle of $5 \lambda / \mathrm{D}_{\text {tel }}$ - equivalent to $9 \mathrm{AU}$ ( Saturn's orbit) at $5 \mu \mathrm{m}$ at $10 \mathrm{pc}$. At this wavelength we probe the younger end of the planet age range giving $\sim 30$ target stars within $10 \mathrm{pc}$.

Dust formation and evolution SPICA will give us an unprecedented window into the key aspects of the dust lifecycle, from the role of stellar winds in the evolution of massive stars to the formation of dust around evolved stars, its processing and evolution in the interstellar medium (ISM), dust destruction in supernova-generated shock waves and regions of massive star formation, through to the formation of proto-planetary disks and the dust seen in comets in our own Solar system as discussed in the previous section. The critical keys to unlocking our understanding of the dust evolutionary cycle are MIR/FIR spectroscopy and the FIR to submillimetre dust SED. The former gives direct information on the dust and ice chemical composition and the latter enables us to constrain the dust size distribution. Previous and near future facilities have either insufficient sensitivity, spatial resolution and/or did not have suitable spectrographic capabilities to really address the evolution of dust from its formation, its subsequent processing in the ISM and its re-emergence in dense star forming nebulae. SPICA will have the perfect combination of spatial and spectral resolution combined with high sensitivity to follow the evolution of dust from the creation of sub-micron particles in stellar atmospheres to the formation of planets.

\subsection{Galaxy evolution: near and far}

One of the most important discoveries of recent years has been of a population of massive, dusty star-forming galaxies that emit a significant fraction of their restframe bolometric luminosity in the FIR. These ultra-luminous and luminous infrared galaxies (ULIRGs) are relatively rare in the local Universe, but are much more common at high-redshift and their integrated luminosity is believed to account for a significant fraction of the Cosmic Infrared Background (CIRB) [19]. Their very existence challenges the expectations from cold dark matter (CDM) cosmological models and it has become clear that serious revisions are needed to either the initial mass function in high-redshift star formation [20], and/or the star formation/AGN feedback processes in massive distant star-forming galaxies [21]. 
SPICA offers a host of critical MIR/FIR diagnostics with which to detect and quantify the star formation and the black-hole accretion components at increasing redshift, thereby following back galaxy evolution. The unprecedented sensitivity, wavelength coverage and spatial resolution of the SPICA instruments will enable the study of the physical conditions in active nuclei, central starbursts, circumnuclear rings, disks, winds and halos in galactic laboratories in the local Universe, as well as comprehensively in sources in the distant Universe. The field of view of the SPICA instruments provides a considerable spatial multiplexing advantage over other facilities, crucial to studies of both the distant Universe, where source densities and clustering means that multiple sources can be viewed in a single footprint, and mapping of local, resolved, galaxies. Photometrically, SPICA has the sensitivity to detect high-luminosity objects out to $z>4$ and Milky Way-type populations out to $z \sim$ 1 , whilst spectroscopically SPICA will characterise the excitation conditions and source physics of the distant dusty galaxy populations resolved in deep photometric Herschel surveys out to $z>3$. In the remainder of this section we illustrate some of the key science questions that SPICA will enable us to address.

The AGN-starburst connection at high-redshift By the launch of SPICA, deep cosmological surveys will have detected many thousands of faint and distant MIR/ FIR galaxies, ranging from sources that dominate the source counts, to those much rarer and more exotic at the highest redshifts. Observational evidence suggests that the properties of the faint MIR/FIR population differ markedly from those of similarly luminous, local infrared galaxies [22]. SPICA will be able to use spectroscopy to constrain many of the key physical properties of the gas in these galaxies such as temperature, density and metallicity, as well as characterising the radiation field so distinguishing the AGN and starburst characteristics of these sources [34]. Figure 5 shows the predicted intensities of a selection of fine-structure emission lines that trace AGN, stellar ionization and PDR regimes, plotted as a function of redshift for three template local objects-M82, NGC1068 and NGC 6240. Sensitivity is key to our ability to probe the distant Universe. With SOAP detectors $\left(5 \sigma-1 \mathrm{~h}\right.$ few $\left.\times 10^{-18} \mathrm{Wm}^{-2}\right)$ it will be possible to detect the very brightest lines in the most exotic objects at high-z; in contrast, with goal sensitivity $(5 \sigma-1 \mathrm{~h}$ few $\times 10^{-19} \mathrm{Wm}^{-2}$ ), not only the brightest MIR line emission (e.g. [NeII]) will be visible from a significant fraction of the SCUBA and Spitzer populations $\left(L_{\mathrm{IR}}>10^{11}\right.$ $L_{\odot}$ ) but also the fainter, but diagnostically important lines of, e.g. [OIV], [NeV] and the rotational transitions of $\mathrm{H}_{2}$. SPICA will provide an important complement to ALMA which will trace the colder, molecular component of the ISM through observations of redshifted $\mathrm{CO}, \mathrm{HCN}$ as well as $[\mathrm{CI}]$ and the longer-wavelength, restframe, FIR cooling lines as they are redshifted into the sub-millimetre waveband.

Observations of intermediate and high-redshift sources have shown that the comoving star formation rate density in the Universe was at least a factor of 10 higher at a redshift of unity [23,24], with more than half of the stars in the universe formed since $z \sim 1$. Previous space-borne spectrometers could barely detect starbursts such as M82 and brighter at intermediate/high redshift, however such objects make up a small fraction of galaxies. With SPICA, for the first time we will be able to characterise the ISM of the complete $z \sim 1$ population, and so follow the evolution as 


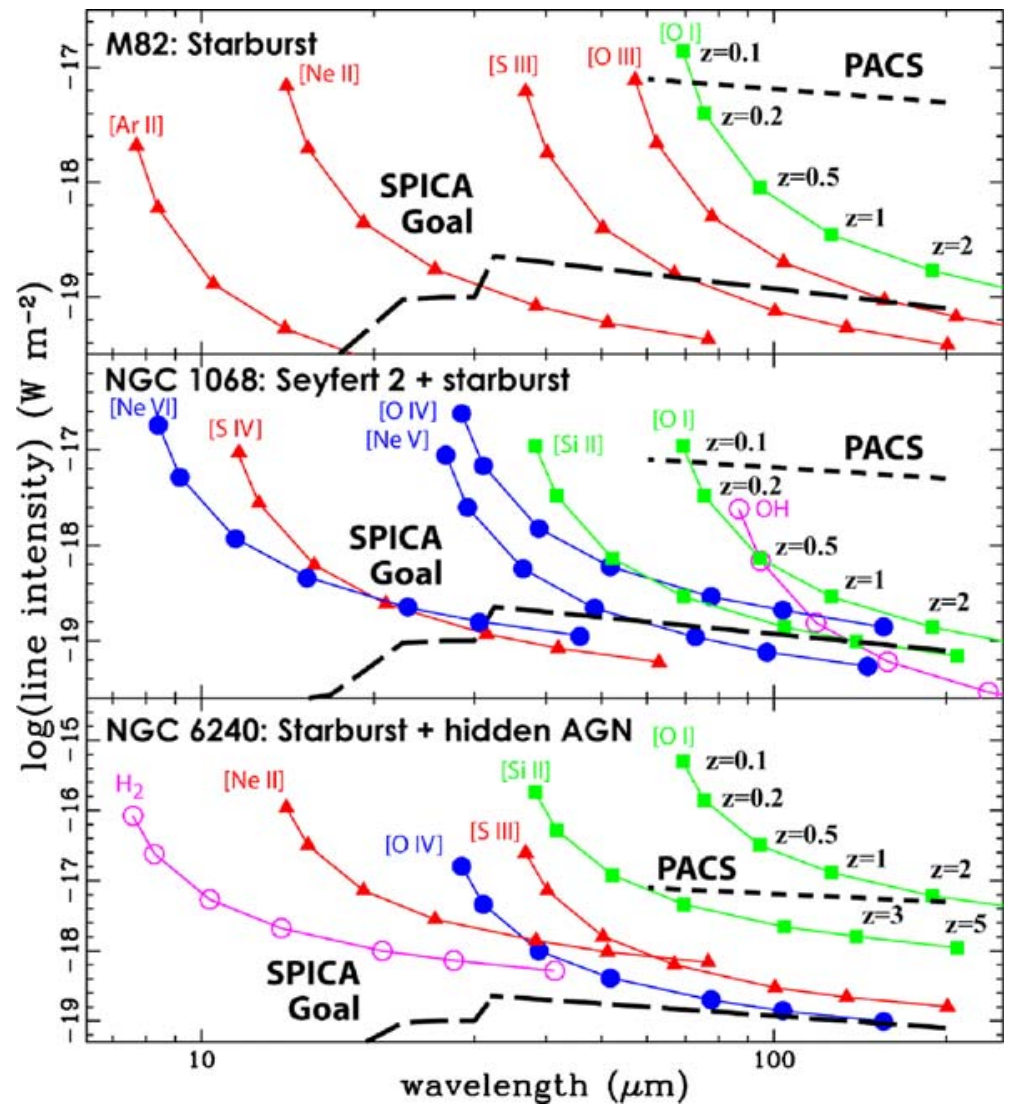

Fig. 5 A plot of intensity vs. redshift of a selection of the key MIR/FIR emission lines visible with SPICA in three archetypical objects: M82 $\left(L_{\mathrm{FIR}} \sim 4 \times 10^{10} L_{\odot}\right)$, NGC1068 $\left(L_{\mathrm{FIR}} \sim 2 \times 10^{11} L_{\odot}\right)$ and NGC6240 $\left(L_{\mathrm{FIR}} \sim 7 \times 10^{11} L_{\odot}\right)$ - in each panel the upper/lower dashed lines denote the $5 \sigma-1 \mathrm{~h}$ sensitivity of HerschelPACS/SPICA respectively

a function of redshift of these ubiquitous galaxies. Galaxies such as our own Milky Way will be easily detectable in the continuum out to $z \sim 1$, whilst the MIR/FIR cooling lines will be visible out to $z \sim 0.5$ where the universal SFR has increased by close to an order of magnitude.

Deep continuum surveys The $70 \mu \mathrm{m}$ confusion limit for SPICA is $\sim 50 \mu \mathrm{Jy}$, a depth that can be achieved with goal detector sensitivity in a few minutes with simultaneous measurements of similar sensitivity in the other two wavebands of the SPICA FIR instrument. Using current assumptions of the intensity of the CIRB at this wavelength, a $70 \mu \mathrm{m}$ confusion-limited survey would resolve more than $90 \%$ of the CIRB over $80 \%$ of the Hubble time $(z \sim 2)$, by detecting galaxies down to the star formation rate (SFR) regime where the UV meets the IR, i.e. around $10 \mathrm{M}_{\mathrm{sol}} /$ year [25]. SPICA will also be sensitive to many galaxies as quiescent as our own $\left(L_{\mathrm{IR}}<10^{10} L_{\mathrm{sol}}\right)$ out to $z \sim 1$, where the cosmic SFR peaks. A $70 \mu \mathrm{m}$ confusionlimited survey will not only provide the best measurement of the SFR in distant 
galaxies, without being affected by e.g. PAHs, but it will also complete the census on the growth of massive black holes by probing the missing population of dustobscured, mostly Compton-thick, AGNs responsible for the unresolved peak of the $\mathrm{X}$-ray background at $30 \mathrm{keV}$.

Both JWST MIRI and SPICA MIR will provide unprecedented angular resolution at MIR wavelengths, $\sim 0.35$ arcsec at the shortest wavelengths, comparable to the angular resolution of hubble space telescope (HST)/near infrared camera and multiobject spectrometer (NICMOS) in the near-IR. At this angular resolution it will be possible to study the morphology of both stellar emission in galaxies out to high $z$, as is now possible at shorter wavelengths with ultra-deep HST/NICMOS observations. Deep MIR extragalactic surveys will be performed by JWST MIRI prior to the launch of SPICA with better sensitivity at the short wavelengths (Fig. 1). However, SPICA, with its much larger field of view and multi-waveband observation capability over four MIR channels ( 5 to $38 \mu \mathrm{m}$ ) will not only map the sky more rapidly at approximately $>15 \mu \mathrm{m}$ (and at comparable speeds at shorter wavelengths; see Fig. 6), but also trace both the stellar (short MIR) and dust components (long MIR) in the same field.

Punching through the traditional FIR confusion limit To resolve the objects making up the CIRB requires the spatial resolution of an interferometer such as FIRI, however, SPICA's combination of spectral imaging and large wavelength coverage provides a novel way to break the traditional confusion limit by adding wavelength as a third dimension to deep, cosmological surveys. Sources can be

Fig. 6 The FOV of 2 of the 4 channels of the SPICA MIR (for further technical details see Fig. 11) are shown overlaid on the GOODS-S (epoch 2) IRAC $8 \mu \mathrm{m}$ image. Ringed objects marked have fluxes of $\mathrm{S}(8 \mu \mathrm{m})>20 \mu \mathrm{Jy}$, the $5 \sigma-1 \mathrm{~h}$ sensitivity limit for detection in SPICA MIR low-resolution spectroscopy. Shown for comparison are JWST FOVs: more than 100 sources can be observed in a single pointing of SPICA-MIR

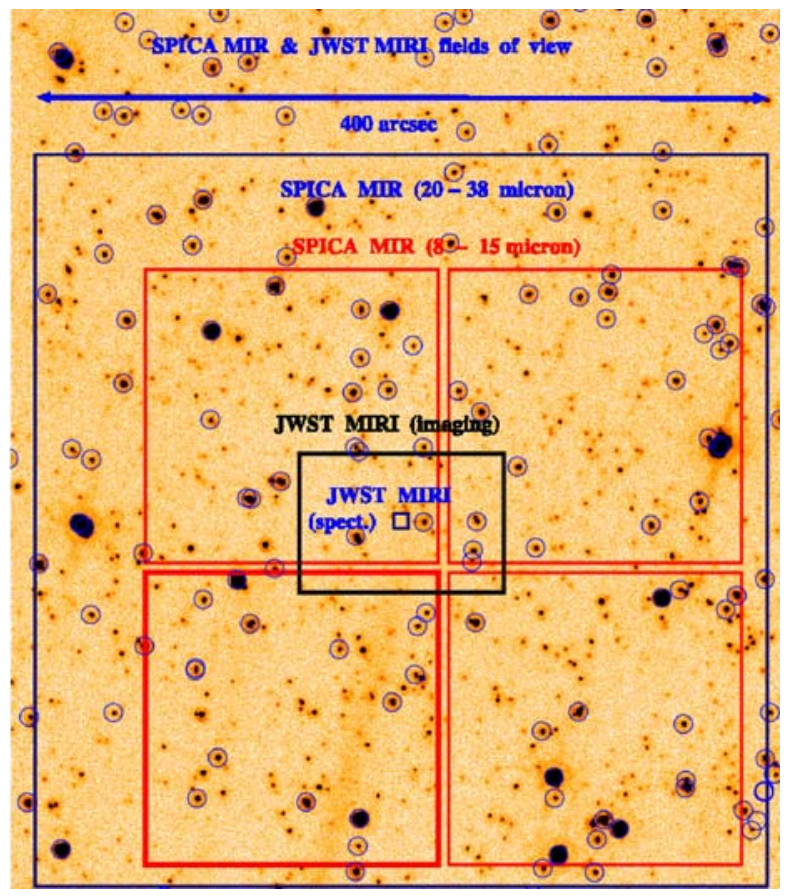


identified by emission from redshifted MIR/FIR cooling lines produced by star formation and AGN activity, rather than by the broad-band signatures of warm dust. Redshifts accurate enough for immediate follow-up ground-based $\mathrm{CO}$ observations (tracing the molecular gas component) can be determined from the detected lines, while line ratios provide a first indication of the nature of the source powering the FIR emission. In simulations of deep, blind spectral line surveys (rms noise level of $0.4 \mathrm{mJy} 100 \mathrm{~h} \mathrm{SOAP} / 10 \mathrm{~h} \mathrm{GOAL}$ at $R \sim 1,000$ ) with SPICA FIR [26] it was possible to detect and determine redshifts of sources brighter than $1 \mathrm{mJy}$ at $120 \mu \mathrm{m}$ out to $z \sim 2.5$ using the five strongest FIR cooling lines (including [OI] at $63 \mu \mathrm{m}$ ), thus probing to a factor of 5 below the $120 \mu \mathrm{m}$ continuum confusion limit. Sources at even higher redshift can be recovered by including MIR emission lines. Simulations suggest that sufficient sources/redshifts can be detected to differentiate between galaxy evolution models, and thus place tight constraints on the origin and history of our own and other galaxies, by observing areas as small as 4 " $\times 4$ ".

Cosmology using low-resolution spectroscopy The rich spectrum of PAH and silicate features found in the MIR of both local [27] and distant galaxies [28] provide an excellent means through which to undertake deep, blind surveys using low-resolution spectroscopy. In the deepest spectra obtained with Spitzer IRS [29], these features can be seen out to $z>2$ in sources that are both optically and MIRfaint $(\mathrm{S}(24 \mu \mathrm{m}) \sim 100 \mu \mathrm{Jy})$ - low-medium resolution spectroscopy $(R \sim 100-200)$ thus provides an excellent means by which to determine source redshifts and measure the physical conditions in sources that would not be detectable using optical or near-IR spectrographs on even the largest ground-based telescopes. The low-resolution modes of the SPICA MIR and FIR instruments will enable largescale spectroscopic surveys of very faint galaxies, down to $\sim 20$ and $\sim 100 \mu \mathrm{Jy}$, respectively. Wide-field, low-resolution spectroscopic surveys with SPICA FIR over an area of the size of the COSMOS field (2 square degrees) with integration time of $1 \mathrm{~h}$ per pointing would provide spectra of $\sim 18,000$ high- $z$ galaxies in $1,800 \mathrm{~h}$. This is more than a factor of 50 times greater than the total number of high- $z$ galaxies observed with Spitzer IRS. Through measurements of the PAH features we will not only be able to directly estimate star formation rates [30], but also to measure redshifts and so start to evaluate the co-moving space density of this dusty population and to constrain the bolometric luminosity function of luminous infrared galaxies.

\subsection{Discovery science}

The science case we have presented in this section illustrates the concrete and definitive progress in our understanding of the Universe that SPICA will allow. However, as with all strides in increased sensitivity or the opening of new wavelength bands, new discoveries are certain once SPICA starts observing. Witness the discoveries made by IRAS, ISO and Spitzer of ULIRGS, crystalline silicates in dust in astrophysical sources and the presence of large amounts of dust in high redshift galaxies. With an increase in sensitivity of another two orders of magnitude 
in the FIR we can expect some real progress in areas where even upper limits will constrain the boundaries of what are, today, rather speculative theories, to wit:

- Constraints on the emission of ground state $\mathrm{H}_{2}$ emission from the first (population III) generation of stars

- The detection of biomarkers in the MIR spectra of exo-planets and/or the primordial material in proto-planetary disks

- The detection of $\mathrm{H}_{2}$ haloes around galaxies in the local Universe

- With sufficient technical development of coronagraphic techniques: the imaging of any planets in the habitable zone in the nearest few stars

- The detection of the far-infrared transitions of PAHs in the interstellar medium. The very large molecules thought to comprise the PAHs, and which give rise to the characteristic features in the MIR, have vibrational transitions in the FIR which are widespread and could be extremely weak [31].

- The direct detection of dust formation in super novae in external galaxies and the determination of the origin of the large amounts of dust in high redshift galaxies

\subsection{SPICA science requirements}

Table 1 summarises the key functional and performance requirements of SPICA that are necessary to achieve the scientific programme described above.

\section{SPICA mission profile}

Overview SPICA is part of the JAXA future science programme and is planned for launch in 2017. The mission follows on both scientifically and technically from the highly successful AKARI mission. High photometric sensitivity observations in the MIR/FIR are made possible thanks to the large $3.5 \mathrm{~m}$ telescope which is actively cooled to below $5 \mathrm{~K}$ to effectively eliminate the non-astronomical photon noise. High spatial

Table 1 Summary of the key functional and performance requirements of SPICA

\begin{tabular}{|c|c|}
\hline Requirement & Comment \\
\hline Spacecraft & $\begin{array}{l}\text { 3-axis stabilized with high pointing accuracy and stability in a thermally benign orbit } \\
\text { which provides all sky access during the course of the mission }\end{array}$ \\
\hline Telescope & $\begin{array}{l}\text { As large a monolithic aperture possible compatible with the launcher fairing }(3.5 \mathrm{~m}) \text {. } \\
\text { Diffraction limited imaging at } 5 \mu \mathrm{m} \text { over a field of view sufficiently large to accommodate } \\
\text { the proposed focal plane instruments. Point spread function sufficiently controlled to } \\
\text { allow pupil mask coronagraphy in the MIR. Temperature }<5 \mathrm{~K} \text { to remove self emission in } \\
\text { the FIR. Stray-light controlled to a level to allow Zodiacal light limited sensitivity. }\end{array}$ \\
\hline $\begin{array}{l}\text { Focal plane } \\
\text { instruments }\end{array}$ & $\begin{array}{l}\text { Full wavelength coverage from } 5 \text { to } 210 \mu \mathrm{m} \text {. Instruments required to provide } \\
\text { Photometric camera function, a spectroscopic function and a coronagraph working } \\
\text { in the MIR range. The instrument instantaneous field of view in both Photometer } \\
\text { and Spectrometer modes to be as large as possible dictated by available spacecraft } \\
\text { resources and technology. Sensitivity as close to background limited as possible. }\end{array}$ \\
\hline Operations & $\begin{array}{l}\text { Sufficient telemetry resources to allow uncompressed science data downlink } \\
\text { ( } \sim 30 \mathrm{~GB} \text { /day) however a strong design driver exists for higher total capacities to } \\
\text { allow downlink of raw detector samples. }\end{array}$ \\
\hline
\end{tabular}


resolution is achieved thanks to the large aperture, monolithic primary mirror and the appropriate tolerances on the telescope and mirror surfaces to achieve diffraction limited performance at $5 \mu \mathrm{m}$.

Launch/orbit The launcher selected for SPICA is the JAXA H2A-202, which is compatible with the overall mission requirements. It launches from the Tanegashima Space Centre and can deliver a payload mass 2,600 kg to L2. The H2A-202 is the smallest variant of the $\mathrm{H} 2 \mathrm{~A}$ and therefore any growth in the payload mass can be accommodated by selecting a larger variant. The payload fairing on the launcher is $ø 4,600 \mathrm{~mm}$ which is compatible with the $3.5 \mathrm{~m}$ telescope and associated thermal baffling. The orbit selected for SPICA is the S-E L2 libration point as it provides a benign and stable thermal environment required to cool the payload to $<5 \mathrm{~K}$ as well as a good instantaneous sky visibility.

Space segment In order to meet the 2017 launch date and keep within the financial constraints of the participating agencies, the key elements of the space segment must rely either on existing technology or on modest development programmes. A nominal lifetime of 5 years is baselined, permitting the science objectives to be met while allowing margin for exploratory science. Sufficient spacecraft consumables for attitude control etc. will be carried to permit 10 years of operations in order to accommodate possible mission extensions.

Ground segment The mission requires a minimum of five hours ground contact per day. The spacecraft and instruments will have sufficient autonomy functions to be tolerant to the loss of ground contact for extended periods of time. The mission will be greatly enhanced by the provision of additional ground contact time to increase the telemetry budget and this might be provided by the use of an ESA ground station.

\subsection{Mission level critical issues}

Spacecraft Thermal design This is the main critical technical issue facing the spacecraft. The main components of this challenge include: (1) the speed and efficiency of the cool down from ambient temperature at launch to the operational cryogenic temperatures and (2) the steady state temperatures and cooler heat loads during nominal operations. Regarding the cool down; the design must account for the actual heat lift capacities of the coolers at different operating temperatures and avoid any pinch points where static heat loads exceed the system cooling capacity. The scientific impact of a non-compliant thermal system will depend on the exact nature of the problem and ranges from degraded performance to loss of function. The thermal design of SPICA has been the subject of much detailed design and analysis [32] and is recognised within the SPICA project as requiring ongoing detailed analysis and verification to ensure that the requirements are met.

AIT System-level test and calibration represents a significant challenge in the SPICA programme. The satellite will be launched warm, followed by a controlled cool down to the operating temperature during the initial phases of the mission. This approach brings several benefits, in particular making the spacecraft simpler and lighter. It does, however 
make it more difficult to simulate a flight-like environment for the spacecraft and payload. One particular challenge is verification of the various payload opto-mechanical budgets under a flight-like thermal and zero-g environment. To address this, an existing JAXA cryogenic test facility is to be upgraded to allow optical testing of the telescope assembly and instrument suite at the operating temperature of $<5 \mathrm{~K}$. Other aspects of the AIT programme are considered to be of a more routine nature.

The SPICA spacecraft The layout of the SPICA spacecraft is schematically illustrated in Fig. 7. The main elements of the spacecraft include the Telescope Assembly (including instrument suite), sunshield/stray-light baffles, solar array and the spacecraft bus. The Telescope Assembly and instrument suite are cooled to $<5 \mathrm{~K}$ with a system of mechanical cryocoolers and passive radiative cooling. This obviates the need for a heavy cryostat and a store of consumable liquid cryogens to provide cooling capacity and means that the total mass of the spacecraft is kept to $2,600 \mathrm{~kg}$ including $180 \mathrm{~kg}$ of propellant. This approach also has the benefit of a warm launch for the payload which makes environmental vibration qualification simpler and opens up the possibility of employing engineering solutions for thermally and mechanically efficient cryogenic structures.

Thermal subsystem Two separate Joule-Thompson (J-T) coolers are used for active cooling of the Telescope Assembly and instruments. The working fluid in both J-T coolers is pre-cooled by a $20-\mathrm{K}$ Stirling cooler which allows an ultimate temperature of $1.7 \mathrm{~K}$ for the ${ }^{3} \mathrm{He} \mathrm{J}-\mathrm{T}$ cooler and $4.5 \mathrm{~K}$ for the ${ }^{4} \mathrm{He} \mathrm{J}-\mathrm{T}$ cooler.

The total $4.5 \mathrm{~K}$ heat load budget for the telescope assembly and instrument suite, including wiring and structural parasitics, radiation and dissipation within the
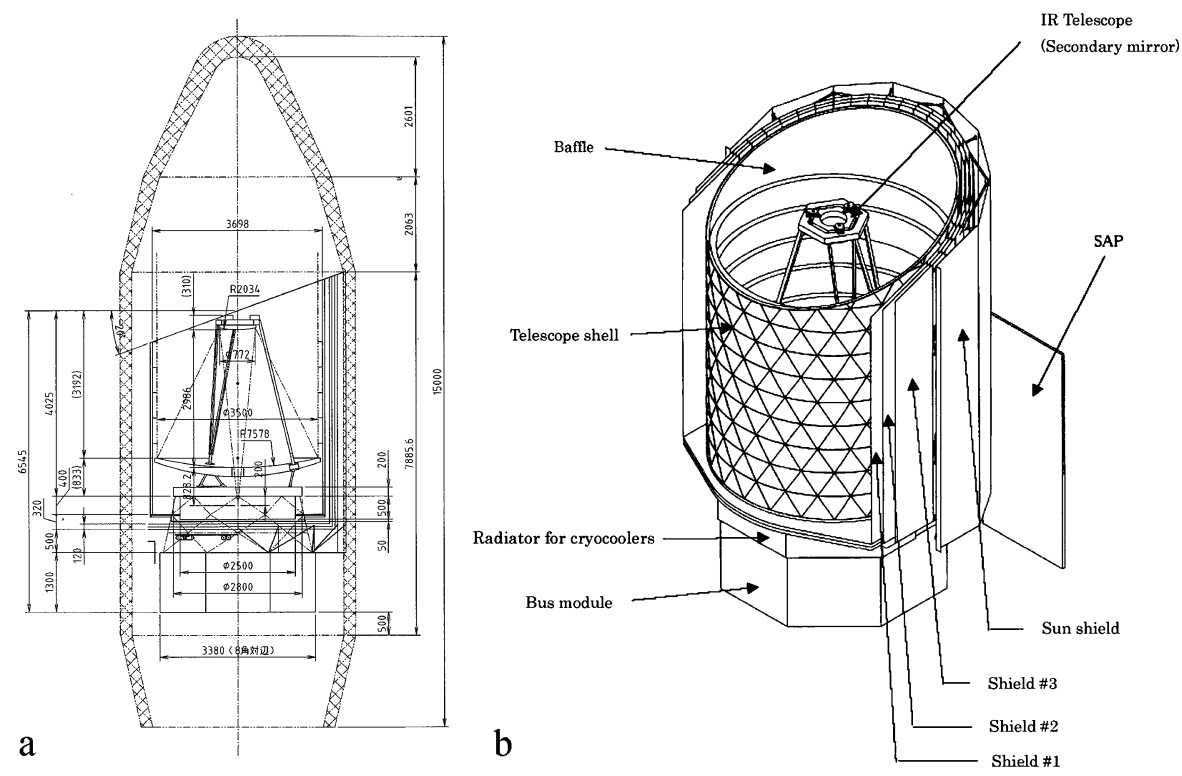

Fig. 7 Architectural layout of the SPICA spacecraft; (a) stowed in the H2A fairing and (b) 3D cut-away view illustrating the thermal stages 
instruments is $28 \mathrm{~mW}$. The development cooler has demonstrated a maximum heat lift capacity of $50 \mathrm{~mW}$ at $4.4 \mathrm{~K}$ (beginning-of-life (BOL) performance) under representative flight conditions with a total primary power consumption of less than $160 \mathrm{~W}$. The heat load budget for the $1.7 \mathrm{~K}$ stage of the ${ }^{3} \mathrm{He} \mathrm{J}-\mathrm{T}$ cooler is $5 \mathrm{~mW}$ which is allocated to cooling the ESI detector subsystem. This cooler has demonstrated a heat lift of $16 \mathrm{~mW} @ 1.7 \mathrm{~K}$ (BOL performance). The high temperature heat load from the coolers is radiated to space with a system of loop heat pipes and radiator panels. The Stirling cooler is based on AKARI heritage and is under development to upgrade the heat lift capacity. Similarly, the J-T coolers are based on an upgrade of the JAXA JEM/SMILES cooler.

A critical aspect of the thermal design is the cool-down of the payload assembly from ambient temperatures at launch to the cryogenic operational temperatures. It is currently baselined to use a Stirling pre-cooler to ensure the payload elements reach a temperature below the J-T coolers pinch point before they are switched on. The current analysis indicates that the telescope will reach operational temperature 170 days after launch.

Power The solar array and power control and distribution system are sized to provide $2.4 \mathrm{~kW}$ (end-of-life (EOL)) of primary power to the instruments and spacecraft subsystems. The main spacecraft bus provides unregulated power to the subsystems at voltages between 32.5 and $52 \mathrm{~V}$.

CDMS The spacecraft Command and Data Management System uses a Spacewire bus for instrument and spacecraft subsystem interfaces. The mass memory has sufficient capacity to buffer all instrument and spacecraft telemetry for a $24 \mathrm{~h}$ period. Sufficient Fault Detection, Isolation and Recovery functions are implemented by the spacecraft and instruments to safe the space segment in the event of an anomaly occurring when the spacecraft is not in direct communication with the Mars orbiter camera.

AOCS The expected pointing error is less than 1" and the stability is less than 30 mas. Higher accuracy will be achieved for the coronagraph by using an internal tip-tilt mirror. Microvibrations from spacecraft mechanisms will be carefully managed and controlled during the satellite development.

\subsection{SPICA payload overview}

Four components of the SPICA payload are described in this section: the SPICA Telescope Assembly (STA); the FIR imaging spectrometer; the MIR imaging spectrometer and the MIR Coronagraph. The division of the functions of the different focal plane instruments is largely dictated by the available detector technologies, which in turn define the types of instrument design that are most appropriate. We concentrate on the elements that will be provided as part of the European hardware contribution to SPICA, namely the telescope and the FIR instrument (ESI), and give brief overviews of the other instruments. Indicative overall resources/interfaces for the instrument suite are summarised in Table 2. 
Table 2 SPICA instrument interface and resource summary

\begin{tabular}{|c|c|c|}
\hline Parameter & $\begin{array}{l}\text { Baseline requirement/ } \\
\text { specification }\end{array}$ & Comments \\
\hline Instrument suite mass & $\begin{array}{l}<200 \mathrm{~kg} \text { total: } 100 \mathrm{~kg} \text { in } \\
\text { focal plane } 100 \mathrm{~kg} \text { within } \\
\text { service module }\end{array}$ & $\begin{array}{l}\text { This is the overall mass of the instruments } \\
\text { including the warm electronics. It is subject } \\
\text { to revision during the SPICA Phase-A study. }\end{array}$ \\
\hline $\begin{array}{l}\text { Cryogenic focal plane } \\
\text { accommodation }\end{array}$ & $ø 2,500 \times 500 \mathrm{~mm}$ height & $\begin{array}{l}\text { The telescope primary focus is at the centre } \\
\text { of this volume. }\end{array}$ \\
\hline $\begin{array}{l}\text { Instrument thermal } \\
\text { interfaces }\end{array}$ & $\begin{array}{l}5 \mathrm{~mW} \text { at } 1.7 \mathrm{~K} \\
10 \mathrm{~mW} \text { at } 2.5 \mathrm{~K} \\
15 \mathrm{~mW} \text { at } 4.5 \mathrm{~K}\end{array}$ & $\begin{array}{l}\text { These values are based on EOL performance } \\
\text { of the spacecraft coolers }\end{array}$ \\
\hline $\begin{array}{l}\text { Power for instrument } \\
\text { analogue and digital } \\
\text { electronics }\end{array}$ & $200 \mathrm{~W}$ & $\begin{array}{l}\text { This is the unregulated power budget for all } \\
\text { the instruments. }\end{array}$ \\
\hline Telemetry budget & 60 GB daily TM & $\begin{array}{l}\text { This corresponds to } \sim 6 \mathrm{MBits} / \mathrm{s} \text { averaged } \\
\text { over a } 24 \mathrm{~h} \text { period and includes the capacity } \\
\text { provided by the ESA Cebreros ground station }\end{array}$ \\
\hline Spacecraft pointing & $\begin{array}{l}\text { Absolute/reconstructed: } 1 " \\
\text { Stability: } 0.03^{\prime \prime} \text { RMS over } \\
60 \mathrm{~s}\end{array}$ & $\begin{array}{l}\text { The absolute pointing requirements are met } \\
\text { after in-orbit calibration of the Attitude and } \\
\text { Orbit Control System }\end{array}$ \\
\hline
\end{tabular}

\subsection{STA}

Description and key characteristics The key features of the STA are a two-mirror on-axis design with a $3.5 \mathrm{~m}$ monolithic primary; an operating temperature below $5 \mathrm{~K}$ with a warm launch and Diffraction limited performance at $5 \mu \mathrm{m}$ over the central portion of the field of view.

The STA comprises the primary and secondary mirrors, the support structure for the mirrors, the telescope optical bench and the structural support structure. A refocusing mechanism is incorporated as a part of the secondary mirror support to compensate for any errors introduced during launch and/or telescope cool-down. A schematic layout of these items is shown in Fig. 8. A list of the key specifications and requirements of the STA is given in Table 3.

The central $30^{\prime}$ diameter of the full field of view is expected to be well corrected for aberrations. The overall $350 \mathrm{~nm}$ root-mean-squared (RMS) transmitted wavefront error (WFE) specification means that diffraction-limited imaging can be achieved with all the SPICA instruments, including the MIR instrument. The compact telescope design requires a relatively large central obscuration $(5 \%)$ due to the relatively large M2 diameter. Additional secondary support struts will add to this entrance pupil obstruction which will cause about a $10 \%$ total loss in throughput. The baseline is to use four struts spaced at $90^{\circ}$ for the M2 support structure. The sidelobes introduced into the point spread function (PSF) by this configuration are highly symmetric and permit the coronagraph to achieve high contrast ratios.

Telescope current heritage and technology readiness level The material baselined for the STA is silicon carbide; chosen for its excellent material properties of high specific strength, stiffness and thermal conductivity, low coefficient thermal expansion, excellent cryogenic stability and the ability to be polished and coated 


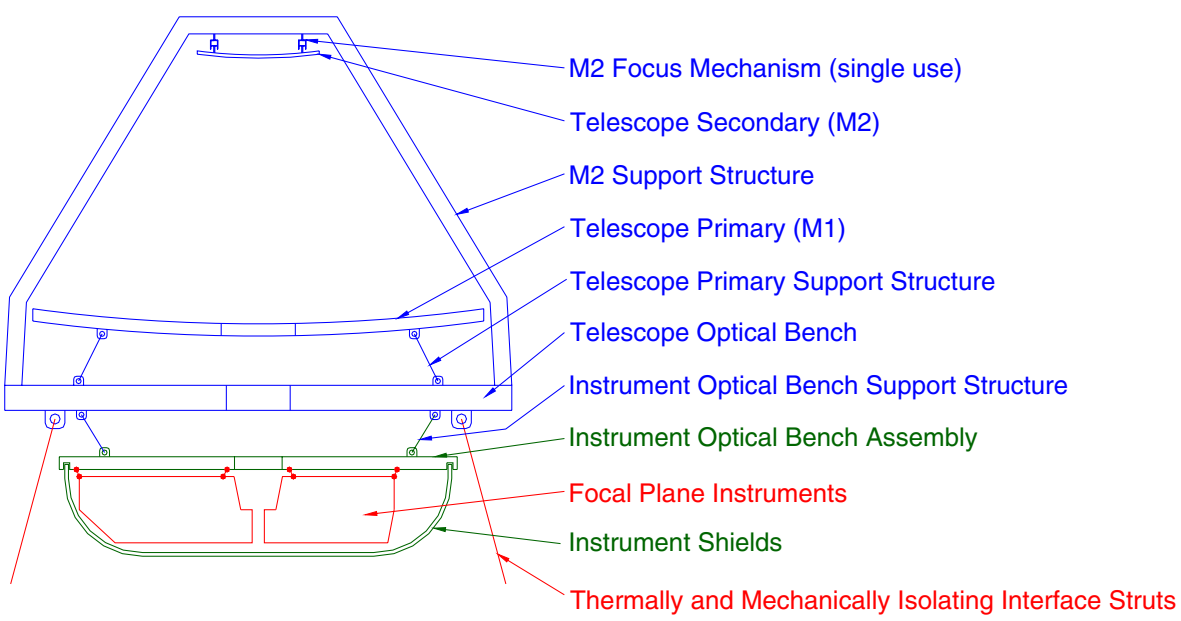

Fig. 8 Schematic representation of the SPICA Telescope Assembly showing proposed ESA funded deliverables in blue, non-ESA deliverables in red with the responsibility for the Instrument Optical Bench Assembly in green

with low emissivity metallic films. This material benefits from the established experience and design heritage in Europe for manufacturing silicon carbide cryogenic space optical elements. In particular the Astrium-France Herschel $(3.5 \mathrm{~m}, 80 \mathrm{~K}, \mathrm{WFE}<6 \mu \mathrm{m})$, ALADIN $(1.5 \mathrm{~m}, \mathrm{WFE}<150 \mathrm{~nm})$ and GAIA $(1.5 \times$ $0.5 \mathrm{~m}, \mathrm{WFE}<20 \mathrm{~nm}$ ) telescopes as well as the ECM-Germany SPICA Breadboard mirror, NIRSpec optical bench and an $800 \mathrm{~mm}, 4 \mathrm{~kg}$ ultra-lightweighted mirror produced under the ESA Future Technology programme. This European heritage is complemented within the Japanese SPICA consortium by the experience with the AKARI telescope and several JAXA sponsored SPICA telescope studies using subscale breadboard models which were used to assess characteristics such as machinability, residual porosity and roughness and surface form variations under cryogenic cycling as well as the optical performance of different silicon carbide materials: $\mathrm{SiC} 100, \mathrm{HB} \mathrm{CeSiC}$ or hybrid $\mathrm{C} / \mathrm{SiC}$ and "reaction sintered" and NT-SiC.

\subsection{FIR imaging spectrometer-ESI ${ }^{1}$}

The baseline optical configuration of ESI is a Mach-Zehnder imaging Fourier Transform Spectrometer. The principal advantages of this type of spectrometer for ESI are the high mapping speed of the FTS due to spatial multiplexing, the ability to incorporate straightforwardly a photometric imaging mode and the operational flexibility to tailor the spectral resolution of the science programme. A grating spectrometer layout has been studied, but due to the limited field of view for the available number of pixels and the difficulty of incorporating a wide field photometer mode, this layout has not been pursued at this time. The principal

\footnotetext{
${ }^{1}$ Note added in proof: The FIR instrument is now named "SAFARI"
} 
Table 3 Key specifications/requirements of the telescope assembly

\begin{tabular}{|c|c|c|}
\hline Parameter & $\begin{array}{l}\text { Baseline specification/ } \\
\text { requirement }\end{array}$ & Comment \\
\hline $\begin{array}{l}\text { Telescope } \\
\text { configuration }\end{array}$ & $\begin{array}{l}\text { 2-mirror on-axis Ritchey- } \\
\text { Chrétien }\end{array}$ & $\begin{array}{l}\text { Compact proven design with wide useable field of } \\
\text { view with good image quality }\end{array}$ \\
\hline $\begin{array}{l}\text { Operating } \\
\text { temperature }\end{array}$ & $<5 \mathrm{~K}$ & $\begin{array}{l}\text { Obtained via passive radiative cooling (to deep } \\
\text { space) }+ \text { mechanical cooler after initial warm launch }\end{array}$ \\
\hline Collecting area & $3.5 \mathrm{~m}$ diameter & $\begin{array}{l}\text { Pupil stop on secondary mirror M2. Clear aperture } \\
>3.4 \mathrm{~m}\end{array}$ \\
\hline $\begin{array}{l}\text { Effective focal } \\
\text { length }\end{array}$ & $18 \mathrm{~m}$ & $\begin{array}{l}\text { Leads to focal ratio of } \sim 5.2 \text { with } 11,46 \% / \mathrm{mm} \text { plate- } \\
\text { scale at telescope focal plane }\end{array}$ \\
\hline $\begin{array}{l}\text { M1-M2 } \\
\text { separation }\end{array}$ & Baseline $3 \mathrm{~m}$ & $\begin{array}{l}\text { To maintain compactness of overall assembly; requires } \\
\text { fast primary mirror }\end{array}$ \\
\hline Back focus & Baseline $0.8 \mathrm{~m}$ & $\begin{array}{l}\text { Minimum distance to accommodate M1 support } \\
\text { structure and focal plane instrument optical bench }\end{array}$ \\
\hline Field of View & $0.5^{\circ}$ diameter & $\begin{array}{l}\text { Large FOV to be shared by instruments taking separate } \\
\text { parts of the field }\end{array}$ \\
\hline Image quality & $\begin{array}{l}\text { Diffraction limited at } 5 \mu \mathrm{m} \\
\text { (at operating temperature) }\end{array}$ & $\begin{array}{l}\text { Strehl ratio }>80 \% \text { at } 5 \mu \mathrm{m} \text { this is equivalent to } \mathrm{WFE}< \\
350 \mathrm{~nm} \text { RMS in central part of the accessible field of } \\
\text { view } \\
\text { Surface roughness requirement of }<20 \mathrm{~nm} \text { RMS }\end{array}$ \\
\hline $\begin{array}{l}\text { Mirror } \\
\text { reflectivity }\end{array}$ & $\begin{array}{l}>97.5 \% \text { above } 30 \mu \mathrm{m} \\
>95 \% \text { below } 30 \mu \mathrm{m}\end{array}$ & This includes particulate contamination effects \\
\hline $\begin{array}{l}\text { Mechanical } \\
\text { accommodation }\end{array}$ & $\begin{array}{l}\text { Mass }<700 \mathrm{~kg} \\
\text { Static design load } 20 \mathrm{~g} \\
\text { First mode }>60 \mathrm{~Hz} \\
\text { Volume } ø 3,500 \mathrm{~mm} \text { cylinder } \\
\text { with } \sim 1: 1 \text { aspect ratio }\end{array}$ & $\begin{array}{l}\text { These figures are the results obtained from ISAS } \\
\text { assessment studies. The mass budget excludes the } \\
\text { mass of the instrument optical bench }\end{array}$ \\
\hline Thermal & $\begin{array}{l}\text { Mirror temperature temporal } \\
\text { stability }<0.25 \mathrm{~K} / \mathrm{h} \\
\text { Max temperature differential } \\
\text { across primary } 0.5 \mathrm{~K}(\mathrm{TBC}) \\
\text { Static heat load } \sim 13 \mathrm{~mW}\end{array}$ & $\begin{array}{l}\text { The temperature stability requirement limits } \\
\text { fluctuations of in-field background emission at long } \\
\text { wavelength. The heat load budget and temperature } \\
\text { differential requirement provides for thermally- } \\
\text { induced local deformations degrading optical } \\
\text { performance }\end{array}$ \\
\hline
\end{tabular}

performance characteristics of ESI, together with comments on scientific performance are outlined in Table 4.

A possible optical layout of the instrument is shown in Fig. 9. This layout incorporates a double fold mirror within the spectrometer mechanism which yields a folding factor of eight. An internal pupil diameter of $\sim 30 \mathrm{~mm}$ and a mirror scan range of $\sim 85 \mathrm{~mm}$ will achieve the goal spectral resolution of $R=\sigma / \Delta \sigma=10,000$ based on $\Delta \sigma=1.2 / 2 \times \mathrm{OPD}$ in the centre of the ESI band.

Detectors for ESI The development of detector technology with suitable performance and architecture for ESI is a critical focus of the instrument development study. A summary of detector performance together with a list of architectural design features and operational constraints/considerations is given in Table 5. In the sections that follow we discuss the development status of each of the detector options.

TES Bolometers TES bolometers require a base temperature in the order of 50$70 \mathrm{mK}$ to achieve the required NEP. This adds significantly to the complexity and mass of the instrument as it necessitates an instrument provided sub-K cooler. The 
Table 4 Key scientific performance characteristics of ESI

\begin{tabular}{|c|c|c|}
\hline Parameter & Specification & Comments/tradeoffs \\
\hline $\begin{array}{l}\text { Instrument } \\
\text { type }\end{array}$ & $\begin{array}{l}\text { Imaging Fourier transform } \\
\text { spectrometer }\end{array}$ & $\begin{array}{l}\text { Allows for a broad-band photometry imaging } \\
\text { mode as well as variable resolution imaging } \\
\text { spectrometry }\end{array}$ \\
\hline $\begin{array}{l}\text { Wavelength } \\
\text { range }\end{array}$ & $\begin{array}{l}30-210 \mu \mathrm{m} \text { : Nominally divided } \\
\text { into } \sim 3-4 \text { sub-bands }\end{array}$ & $\begin{array}{l}\text { Short wavelength limit defined by overlap with } \\
\text { MIR instrument; long wavelength limit defined } \\
\text { by the [NII] } 206 \mu \mathrm{m} \text { line. Division of the spectral } \\
\text { range into the different bands depends on the } \\
\text { final detector technology selection }\end{array}$ \\
\hline $\begin{array}{l}\text { Instantaneous } \\
\text { field of view }\end{array}$ & $2^{\prime} \times 2^{\prime}$ & $\begin{array}{l}\text { Derived from a science requirement to image } \\
\text { diffuse emissions and give a large spatial } \\
\text { multiplexing advantage }\end{array}$ \\
\hline $\begin{array}{l}\text { Angular } \\
\text { resolution }\end{array}$ & $\begin{array}{l}\text { Diffraction limited performance } \\
\text { above } 40 \mu \mathrm{m}\end{array}$ & $\begin{array}{l}\text { Places constraints on ESI optics. The telescope is } \\
\text { specified to be diffraction limited at } 5 \mu \mathrm{m}\end{array}$ \\
\hline Pixel spacing & $\mathrm{F} \lambda / 2$ at the centre of each band & Critical image sampling \\
\hline $\begin{array}{l}\text { Spectral } \\
\text { resolution }\end{array}$ & $\begin{array}{l}\text { Photometric imaging: } R<10 \\
\text { Low resolution mode: } R \sim 100 \\
\text { Medium resolution mode: } R \sim 2,000 \\
\text { at } 100 \mu \mathrm{m} \\
\text { High resolution mode: } R \sim 10,000 \\
\quad(\text { goal } 100 \mu \mathrm{m})\end{array}$ & $\begin{array}{l}\text { To achieve the stated medium resolution } \\
\text { requirement the required optical path difference is } \\
140 \mathrm{~mm} \text {. It is expected that the goal resolution of } \\
10,000 \text { will be met within a restricted field of } \\
\text { view }\end{array}$ \\
\hline Sensitivity & $\begin{array}{l}\text { Broad band photometry }<50 \mu \mathrm{Jy} \\
(5 \sigma-1 \mathrm{~h}) \\
\text { Medium resolution line sensitivity } \\
<10 \times 10^{-20} \mathrm{~W} / \mathrm{m}^{2}\end{array}$ & Required detector NEP $\sim 1 \times 10^{-19} \mathrm{~W} / \sqrt{ } \mathrm{Hz}$ \\
\hline Dynamic range & $>1,000$ within a single field of view & $\begin{array}{l}\text { A supplementary requirement exists to not saturate } \\
\text { the detectors in high spectral resolution on a } \\
\text { bright source }\end{array}$ \\
\hline
\end{tabular}

justifications for retaining the design option of TES bolometers detectors with this extra level of complexity are that there are very good prospects for achieving the ESI detector goal NEP within the schedule constraints of SPICA with TES detectors, the spectral response of the TES gives good access to the wavelength band around $40 \mu \mathrm{m}$ where photoconductors perform poorly and the effects of cosmic ray hits and non-linear response are more benign for bolometers compared with photoconduc-

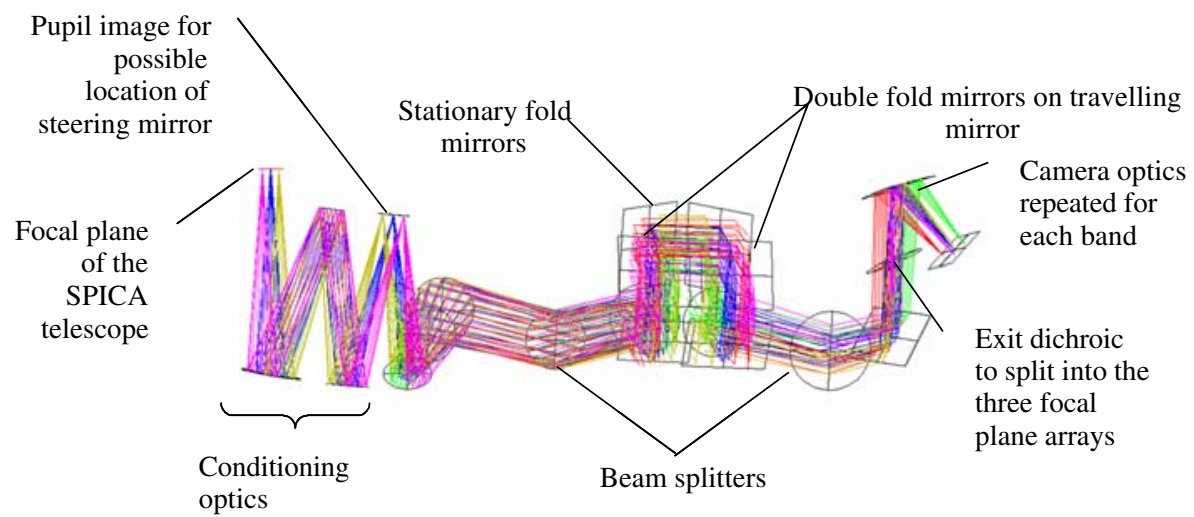

Fig. 9 Possible ESI optical layout. Note: only one arm of the Mach-Zehnder FTS shown for clarity 
Table 5 ESI Detector options

\begin{tabular}{|c|c|c|}
\hline & Option-A TES bolometer & Option-B photoconductor \\
\hline \multirow[t]{3}{*}{ Description } & Band $A=30-57 \mu \mathrm{m}$ & $\begin{array}{l}\text { Band } A=27-37 \mu \mathrm{m}: \text { monolithic Si:Sb } \\
\text { BIB array }\end{array}$ \\
\hline & Band $B=57-106 \mu \mathrm{m}$ & $\begin{array}{l}\text { Band } B=45-110 \mu \mathrm{m} \text { : unstressed monolithic } \\
\text { Ge:Ga }\end{array}$ \\
\hline & Band $\mathrm{C}=106-210 \mu \mathrm{m}$ & $\begin{array}{l}\text { Band } C=110-210 \mu \mathrm{m}: \text { array: stressed } \\
\text { Ge:Ga }\end{array}$ \\
\hline NEP & $\sim 1 \times 10^{-19} \mathrm{~W} / \sqrt{ } \mathrm{Hz}$ & a few $\times 10^{-18} \mathrm{~W} / \sqrt{\mathrm{Hz}}$ \\
\hline \multirow{4}{*}{$\begin{array}{l}\text { Pixel (array) } \\
\text { size }\end{array}$} & Pixel pitch $\sim 2.5 \mathrm{~mm}$ & \\
\hline & Band $A=64 \times 64 \mathrm{Px} \geq 160 \times 160 \mathrm{~mm}$ & Band $\mathrm{A}=256 \times 256 \mathrm{Px} \geq 12.8 \times 12.8 \mathrm{~mm}$ \\
\hline & Band $B=38 \times 38 \mathrm{Px} \geq 95 \times 95 \mathrm{~mm}$ & Band $\mathrm{B}=32 \times 32 \mathrm{Px} \geq 115 \times 115 \mathrm{~mm}$ \\
\hline & Band $\mathrm{C}=20 \times 20 \mathrm{Px} \geq 50 \times 50 \mathrm{~mm}$ & Band $\mathrm{C}=32 \times 32 \mathrm{Px} \geq 115 \times 115 \mathrm{~mm}$ \\
\hline Readout & $\begin{array}{l}\text { Frequency division multiplexed. } \\
\text { Series SQUID array cold } \\
\text { amplification }\end{array}$ & $\begin{array}{l}\text { Direct Injection (DI) circuit cold readout of } \\
\text { photoelectric current }\end{array}$ \\
\hline $\begin{array}{l}\text { Wavelength } \\
\text { coverage }\end{array}$ & $30-210 \mu \mathrm{m}$ & $\begin{array}{l}30-37+45-206 \mu \mathrm{m} \\
\text { Poor performance over "missing octave" }\end{array}$ \\
\hline $\begin{array}{l}\text { Dynamic } \\
\text { range }\end{array}$ & $\begin{array}{l}\text { Fundamentally linked to NEP and } \\
\text { limited to } \sim 10,000 \text { at } 1 \times 10^{-19} \mathrm{~W} / \sqrt{ } \mathrm{Hz}\end{array}$ & $\begin{array}{l}\text { Adjustable via switchable integrating } \\
\text { capacitor and setting of the integration } \\
\text { period }\end{array}$ \\
\hline Linearity & $\begin{array}{l}\text { Good linearity with feedback to the } \\
\text { readout SQUIDs }\end{array}$ & $\begin{array}{l}\text { Ge:Ga detectors have multiple time constants } \\
\text { which gives rise to frequency dependant } \\
\text { detector response which needs sophisticated } \\
\text { characterization and calibration }\end{array}$ \\
\hline $\begin{array}{l}\text { Cosmic ray } \\
\text { susceptibility }\end{array}$ & $\begin{array}{l}\text { Absorber has low cross section and } \\
\text { detector recovers in several time } \\
\text { constant }\end{array}$ & $\begin{array}{l}\text { Relatively high cross-sectional area gives } \\
\text { rise to high hit rate. Hits change } \\
\text { responsivity but calibration restored with } \\
\text { curing techniques }\end{array}$ \\
\hline Time constant & $\tau_{c} \sim 10 \mathrm{~ms}$ & $\sim 10 \mathrm{~ms}$ for $\mathrm{DI}$ \\
\hline $\begin{array}{l}\text { Base } \\
\text { operating } \\
\text { temperature }\end{array}$ & $100-\mathrm{mK}$ for detector elements & $1.7-4.5 \mathrm{~K}$ for detector elements \\
\hline $\begin{array}{l}\text { Heritage } \\
\text { summary }\end{array}$ & $\begin{array}{l}\text { X-ray TES arrays and read-out electronics } \\
\text { are under extensive development by } \\
\text { SRON for XEUS and at JAXA (ISAS) } \\
\text { for NEXT. IR TES detectors are used } \\
\text { on the GBT and SCUBA-2 }\end{array}$ & $\begin{array}{l}\text { ESI detectors would be an evolution of the } \\
\text { Herschel/PACS and SPITZER MIPS } \\
\text { detectors. AKARI FIS uses Ge:Ga stressed } \\
\text { and unstressed detectors }\end{array}$ \\
\hline TRL & 4 & $5-6$ \\
\hline
\end{tabular}

TRL Technology readiness level

tors. A key disadvantage of bolometer detectors compared with photoconductors is the limited dynamic range. Despite this, the overall instrument dynamic range requirements can be met by employing other design elements to enable both faint and bright source observation. Prototype detectors adapted to FIR requirements have been fabricated and tested by Netherlands Institute for Space Research (SRON), Netherlands and Cardiff University, UK (Fig. 10). SRON have successfully tested a device at $205 \mathrm{mK}$ and the results indicate that an $\mathrm{NEP} \sim 4 \times 10^{-19} \mathrm{~W} / \sqrt{\mathrm{Hz}}$ would be obtained for a system operating at $100 \mathrm{mK}$.

Sub-K cooler A hybrid ${ }^{3} \mathrm{He}$ sorption cooler/Adiabatic Demagnetisation Refrigerator concept has been proposed for ESI which can provide continuous cooling power to the detector system below $100 \mathrm{mK}$. This system has the key advantage of being very 

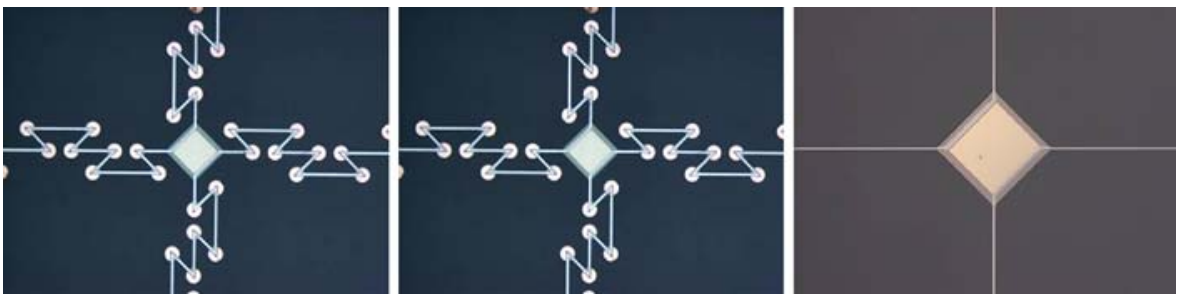

Fig. 10 Various prototype ESI detectors tested at SRON. The detector on the right demonstrated close to the goal performance

compact and light-weight when compared with other systems. One of the key challenges of this architecture is limiting the heat rejected by the instrument cooler to the spacecraft cooler during recycle. Service des Basses Temperatures du Commissariat à l'Energie Atomique are currently carrying out a strategic development programme for such cooler systems for future space science experiments. The most recent tests have achieved a base temperature of $48 \mathrm{mK}$ and successful regulation at $50 \mathrm{mK}$. ISAS also have flight heritage with ADR coolers and have achieved a bath temperature of $60 \mathrm{mK}$ in orbit.

Photoconductors/Si-Bolometers The heritage for the photoconductor detector systems is based on direct experience gained from Herschel, AKARI and indirectly from SPITZER for the Si:Sb technology. The ESI consortium have successfully tested stressed Ge:Ga detectors suitable for ESI to gain information on the behaviour of the devices under very low stray-light conditions and under $\mathrm{p}+$ irradiation. The Belgian Interuniversity Microelectronics Centre are currently developing a Direct Injection amplifier application specific integrated circuit (ASIC) based on $0.35 \mu \mathrm{m}$ technology to be mounted directly on the photoconductor crystal suitable for use in ESI (i.e. very low dissipation/noise/dark current/well depth). It is expected that a prototype of this ASIC will be demonstrated during the summer of 2008.

\subsection{MIR Camera and spectrometer}

The SPICA MIR instrument has two basic modes; a wide-field imaging camera mode and a spectrometer mode. Si:As detectors are used for $\lambda<27 \mu \mathrm{m}$ and $\mathrm{Si}: \mathrm{Sb}$ detectors for $\lambda>26 \mu \mathrm{m}$. A tip-tilt mirror is shared with the Coronagraph to correct fine pointing errors.

Camera mode The wide-field camera has four channels to cover the wavelength range $(5$ to $38 \mu \mathrm{m})$. The pixel scale of the imager $\left(P_{\mathrm{FOV}}\right)$ is optimized for the shortest wavelength $(\lambda \mathrm{s})$ at each channel, that is $P_{\mathrm{FOV}}=\lambda \mathrm{s} / \mathrm{D} / 3$. The imager has several band pass filters and a grism for low resolution objective spectroscopy $(R \sim 200)$. Specifications of the imager are given in Table 6.

The longest wavelength of each channel must be shorter than the twice of the shortest wavelength to avoid higher order mixing while using the grism. Through the use of dichroic beam splitters, the FOV of Channel 1 is placed within that of 
Table 6 Specifications for the SPICA MIR imager

\begin{tabular}{lllll}
\hline Channel & $\begin{array}{c}\text { Wavelength } \\
\text { coverage }(\mu \mathrm{m})\end{array}$ & Pixel FOV & Total FOV & Detector \\
\hline 1 & $5-9$ & $0.098 "$ & $100 "($ Optional 300") & Si:As $1 \times 1$ k (Optional $3 \times 3$ mosaic) \\
2 & $8-15$ & $0.16 "$ & $160 "($ Optional 320") & Si:As $1 \times 1$ k (Optional 2 2 mosaic) \\
3 & $14-27$ & $0.28 "$ & $280 "$ & Si:As $1 \times 1 \mathrm{k}$ \\
4 & $20-38$ & $0.39 "$ & $400 "$ & Si:Sb $1 \times 1 \mathrm{k}$ \\
\hline
\end{tabular}

Channel 3 and the FOV Channel 2 is placed within the FOV of Channel 4. A possible extension of the spatial coverage of the Channel 1 and $2 \mathrm{FOVs}$ is under assessment - this is indicated as an option in Table 6 and Fig. 11.

Spectrometer mode The MIR spectrometer is a long slit grating spectrometer with an Integral Field Unit. It has four channels to cover the wavelength range. In the shorter wavelength region $(\lambda<15 \mu \mathrm{m})$ an immersion grating is used to achieve a spectral resolution of $R \sim 30,000$. The slit width of each channel is tailored for the longest wavelength to avoid diffraction losses. The slit length and number of slices is limited by the number of pixels and wavelength coverage at each band. Consideration is being given to the provision of high resolution Fabry-Perots $(R \sim 30,000)$ to cover the 17 and $28 \mu \mathrm{m} \mathrm{H}_{2}$ lines. The specifications for the spectrometer are given in Table 7. The instrument will be developed by a Japanese/South Korean consortium.

\subsection{MIR Coronagraph}

A coronagraph instrument is proposed to carry out high contrast observations by suppressing the side lobes of the PSF of a bright source (i.e. a star) enabling observation of companion objects (e.g., planets and proto-planetary disks). The instrument wavelength range is $5-27 \mu \mathrm{m}$ with a possible extension to $3.5-5 \mu \mathrm{m}$. The instrument has two observation modes; imaging and $R \sim 200$ spectroscopy. The key specifications of the SPICA coronagraph and a schematic view of the optical layout are shown in Fig. 12. The contrast requirement is $10^{6}$ to detect exo-planets directly

Fig. 11 Focal plane allocation for the SPICA MIR imager

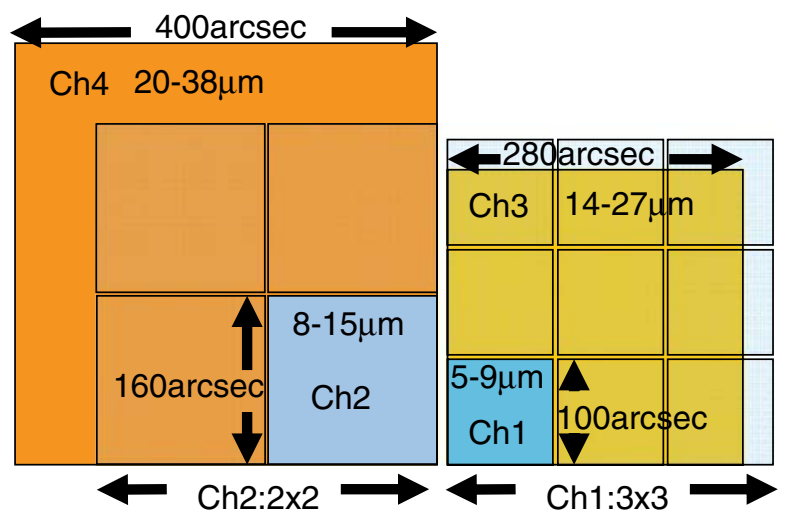


Table 7 Specifications for the SPICA MIR spectrometer

\begin{tabular}{lllllll}
\hline Channel & $\begin{array}{l}\text { Wavelength } \\
\text { coverage }(\mu \mathrm{m})\end{array}$ & $\begin{array}{l}\text { Slit width } \\
\left(2 \times P_{\text {FOV }}\right)\end{array}$ & $\begin{array}{l}\text { Slit } \\
\text { length }\end{array}$ & $\begin{array}{l}\text { Number } \\
\text { of slices }\end{array}$ & $\begin{array}{l}\text { Resolving } \\
\text { power }\end{array}$ & Detector \\
\hline 1 & $5-9$ & $0.53 ”$ & $2.7 ”$ & 3 & 30,000 & $\begin{array}{c}\text { Si:As } 1 \times \\
1 \mathrm{k}\end{array}$ \\
2 & $8-15$ & $0.88^{\prime \prime}$ & $4.4 ”$ & 3 & 30,000 & $\begin{array}{c}\text { Si:As } 1 \times \\
1 \mathrm{k}\end{array}$ \\
3 & 14 S-25 & $1.5 \%$ & $20^{\prime \prime}$ & 5 & 6,000 & $\begin{array}{c}\text { Si:As } 1 \times \\
1 \mathrm{k} \\
\text { Si:Sb } 1 \times \\
1 \mathrm{k}\end{array}$ \\
\hline
\end{tabular}

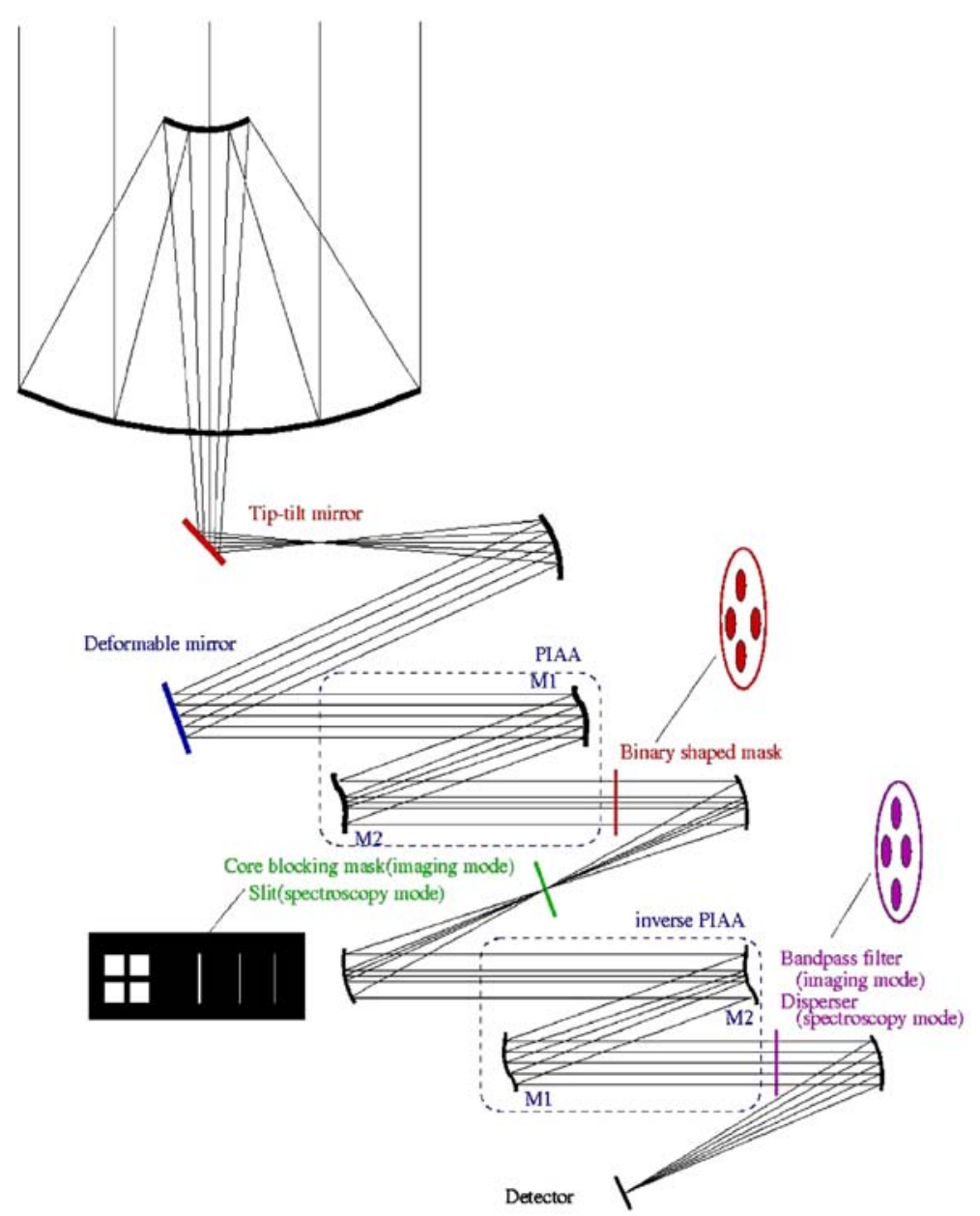

Fig. 12 Schematic optical layout of the Coronagraph and key specifications 
in the MIR. Realising such high contrast is very challenging and we plan to develop two coronagraph methods. The first uses a binary shaped pupil coronagraph, which is regarded as the most simple and robust approach. Laboratory demonstrations of this method have already been successfully done with visible light and a $10^{7}$ contrast has been confirmed in the experiment. The second method uses a hybrid technique of phase induced amplitude apodisation and binary shaped pupil. The advantage of the hybrid solution over the shaped pupil method is that the inner working angle is reduced from $\sim 3.5 \lambda / \mathrm{D}$ to $<2 \lambda / \mathrm{D}$. Another advantage of the hybrid solution is an improvement in throughput from $\sim 30 \%$ for the shaped pupil only method to $\sim 80 \%$ for the hybrid method. Although the hybrid solution requires more complicated optics than the shaped pupil mask only method, a contrast better than $10^{6}$ has been experimentally demonstrated with visible light. The instrument will use an adaptive tip-tilt mirror for fine pointing corrections and final correction of the telescope and instrument input optics WFE will be carried out with a deformable mirror at the input to the coronagraph. The Coronagraph is being developed by a Japanese consortium.

\subsection{BLISS, a proposed US-built grating spectrometer}

A US group centred at JPL is proposing a moderate-resolution compact FIR to sub$\mathrm{mm}$ grating spectrometer, BLISS, for SPICA [33]. BLISS is envisioned as a sensitive suite of grating spectrometers optimized for broadband spectroscopy of distant galaxies. This sensitive point-source capability will complement the core spectral imaging capability of ESI by extending the coverage to longer wavelengths and enhancing the sensitivity for point source spectroscopy. The combination will make SPICA an even more powerful probe of galaxy evolution in the early universe.

\section{Summary and mission status}

Following on from the highly successful Japanese infrared survey mission Akari, SPICA is planned for launch by JAXA on the H2A launcher in 2017 with a nominal five year mission lifetime orbiting at L2. It will feature a cold 3.5-m telescope with the telescope and instruments cooled to $<5 \mathrm{~K}$ using a combination of high reliability mechanical coolers and passive radiative cooling; giving a long lifetime and low launch mass for the mission. At least three focal plane instruments are proposed: an MIR instrument with broadband imaging and spectrographic capabilities to be developed by a Japanese/South Korean consortium; an MIR coronagraph to be developed by Japanese institutes and a (nationally funded) FIR imaging spectrometer to be developed primarily in Europe/Canada with possible contributions from Japan and the USA (National Aeronautics and Space Administration). A fourth instrument giving high sensitivity point source spectroscopy up to sub-mm wavelengths may also be provided by US groups. The combination of a cold telescope and sophisticated instruments will give astronomers unprecedented access to the mid to long wavelength infrared for many years to come and help solve some of the most difficult problems in astrophysics associated with the origin of planets and galaxies and the history of star formation in the Universe. 
We are pleased to report in this paper that SPICA has been selected as one of the candidate missions for further study as part of the ESA Cosmic Vision process.

\section{References}

1. Neugebauer, G., et al.: ApJ. 278, L1 (1984)

2. Cameron, R.M., et al.: Appl. Opt. 10, 2011 (1971)

3. Kessler, M.F., et al.: A\&A 315, L27 (1996)

4. Werner, M.W., et al.: ApJS. 154, 1 (2004)

5. Matsumoto, T.: Proc. SPIE 4013, 47 (2000)

6. Pilbratt, G.L.: Proc. SPIE 5487, 401 (2004)

7. Gardner, J.P., et al.: Proc. SPIE 6265, 17 (2006)

8. Swinyard, B.M., et al.: Proc. SPIE 5487, 413 (2004)

9. ESO: http://www.eso.org/projects/alma/science/bin/sensitivity.html (2007). Accessed June 2007

10. Griffin, M.J., et al.: Appl. Opt. 41, 6543 (2002)

11. Holland, W., et al.: Proc. SPIE 6275, 67 (2006)

12. de Korte, P.A.J., et al.: Proc. SPIE 6266, $1 \mathrm{Z}$ (2006)

13. Malfait, K., et al.: A\&A 345, 181 (1999)

14. Nagasawa, M., et al.: Protostars and Planets V, p. 639. Univeristy of Arizona Press, Tucson (2007)

15. Warren, S.: Appl. Opt. 23, 1206 (1984)

16. Marsh, K.A., et al.: ApJ 646, L77 (2006)

17. Burrows, A., et al.: ApJ 609, 407 (2004)

18. Burrows, , et al.: ApJ 491, 856 (1997)

19. Dole, H., et al.: A\&A 451, 417 (2006)

20. Baugh, C., et al.: 2005 MNRAS 356, 1191 (2005)

21. Granato, G.L., et al.: ApJ 600, 580 (2004)

22. Pope, A., et al.: MNRAS 370, 1185 (2006)

23. Madau, P., et al.: MNRAS 282, 347 (1996)

24. Heavens, A., et al.: Nature 428, 625 (2004)

25. Lagache, , et al.: ApJS 154, 112 (2004)

26. Clements, D.L., et al.: A\&A 465, 125 (2007)

27. Smith, J.D.T., et al.: ApJ 656, 770 (2007)

28. Yan, L., et al.: ApJ 628, 604 (2005)

29. Teplitz, H.I., et al.: ApJ 659, 941 (2007)

30. Tielens, A.G.G.M., et al.: Conf. Proc. PASP 323, 135 (2004)

31. Mulas, G., et al.: A\&A 460, 93 (2006)

32. Sugita, H., et al.: Cryogenics 46, 149 (2006)

33. Bradford, C.M.: Proc. SPIE 6265, $1 \mathrm{X}$ (2006)

34. Spinoglio, L., Di Giorgio, A.M., Saraceno, P.: Adv. Sp. Res. 40, 684 (2007) 